\title{
Olfactory bulb proteome dynamics during the progression of sporadic Alzheimer's disease: identification of common and distinct olfactory targets across Alzheimer-related co- pathologies
}

\author{
María Victoria Zelaya ${ }^{1}$, Estela Pérez-Valderrama ${ }^{1}$, Xabier Martínez de Morentin ${ }^{1}$, \\ Teresa Tuñon ${ }^{2}$, Isidro Ferrer ${ }^{3}$, María Rosario Luquin ${ }^{4}$, Joaquín Fernandez- \\ Irigoyen $^{1, *}$ and Enrique Santamaría ${ }^{1, *}$ \\ ${ }^{1}$ Proteomics Unit, Clinical Neuroproteomics Group, Navarrabiomed, Fundación Miguel Servet, Proteored-ISCIII, Instituto de \\ Investigación Sanitaria de Navarra (IdiSNA), Pamplona, Spain \\ 2 Pathological Anatomy Department, Navarra Hospital Complex, Instituto de Investigación Sanitaria de Navarra (IdiSNA), \\ Pamplona, Spain \\ ${ }^{3}$ Institut de Neuropatologia, IDIBELL-Hospital Universitari de Bellvitge, Universitat de Barcelona, L'Hospitalet de Llobregat, \\ Spain, CIBERNED (Centro de Investigación Biomédica en Red de Enfermedades Neurodegenerativas), Spain \\ ${ }^{4}$ Laboratory of Regenerative Therapy, Department of Neurology and Neuroscience Division, Centre for Applied Medical \\ Research (CIMA), University of Navarra, Instituto de Investigación Sanitaria de Navarra (IdiSNA), Pamplona, Spain \\ * These authors share senior authorship
}

Correspondence to: Enrique Santamaría, email: esantamma@navarra.es

Keywords: Alzheimer, neurodegeneration, olfactory bulb, proteomics, Gerotarget

Received: July 16, $2015 \quad$ Accepted: September 30, $2015 \quad$ Published: October 28, 2015

This is an open-access article distributed under the terms of the Creative Commons Attribution License, which permits unrestricted use, distribution, and reproduction in any medium, provided the original author and source are credited.

\section{ABSTRACT}

Olfactory dysfunction is present in up to $90 \%$ of Alzheimer's disease (AD) patients. Although deposition of hyperphosphorylated tau and $\beta$-amyloid substrates are present in olfactory areas, the molecular mechanisms associated with decreased smell function are not completely understood. We have applied mass spectrometry-based quantitative proteomics to probe additional molecular disturbances in postmortem olfactory bulbs (OB) dissected from AD cases respect to neurologically intact controls $(n=20$, mean age 82.1 years). Relative proteome abundance measurements have revealed protein interaction networks progressively disturbed across AD stages suggesting an early imbalance in splicing factors, subsequent interrupted cycling of neurotransmitters, alteration in toxic and protective mechanisms of $\beta$-amyloid, and finally, a mitochondrial dysfunction together with disturbance in neuron-neuron adhesion. We also present novel molecular findings in the $O B$ in an autopsy cohort composed by Lewy body disease (LBD), frontotemporal lobar degeneration (FTLD), mixed dementia, and progressive supranuclear palsy (PSP) cases $(n=41$, mean age 79.7 years). Olfactory mediators deregulated during the progression of AD such as Visinin-like protein 1, RUFY3 protein, and Copine 6 were also differentially modulated in the OB in LBD, FTLD, and mixed dementia. Only Dipeptidyl aminopeptidase-like protein 6 showed a specific down-regulation in AD. However, no differences were observed in the olfactory expression of this protein panel in PSP subjects. This study demonstrates an olfactory progressive proteome modulation in $A D$, unveiling crossdisease similarities and differences especially for specific proteins involved in dendritic and axonic distributions that occur in the $O B$ during the neurodegenerative process. 


\section{INTRODUCTION}

The olfactory bulb (OB) is the first site for the processing of olfactory information in the brain and its deregulation is associated with neurodegenerative disorders (NDs) [1, 2]. In many cases, the olfactory deficit is an early event of these diseases being considered as a premotor sign of neurodegeneration and consequently, a reliable premature marker of NDs [3]. It has been suggested that the potential origins of olfactory dysfunction may be the depositions and inclusions of Tau, $\beta$-amyloid, and $\alpha$-synuclein proteins in the OB, olfactory tract, and olfactory cortex $[2,4,5]$. Moreover, the presence and severity of hyperphosphorylated Tau, $\beta$-amyloid, and $\alpha$-synuclein pathology in the OB reflects the presence and severity of respective pathologies in other brain regions [1]. On the other hand, the reduction of the cholinergic centrifugal inputs to the $\mathrm{OB}$ and the increased number of the dopaminergic cells observed in the OB region $[4,6]$ have also been suggested as potential origins of smell loss.

Although olfactory involvement may also appear in healthy non-demented elderly subjects [7], olfactory dysfunction is present in up to $90 \%$ of $\mathrm{AD}$ patients [1]. Some studies suggest that olfactory dysfunction is an early event of $\mathrm{AD}$, preceding the appearance of typical $\mathrm{AD}$ symptoms, such as memory loss, and dementia. Neuropathological studies have pointed out that olfactory centres are involved in early Braak stages [8], and OB pathology correlates with cortical AD pathology [2, 9, 10]. An OB atrophy and a significant reduction in olfactory performance have been detected in $\mathrm{AD}$ respect to control subjects using MRI and PET technologies [11, 12]. In view of these data, an in depth biochemical characterization of the neurodegeneration that occurs in the $\mathrm{OB}$ is mandatory as a first step for understanding early smell impairment in $\mathrm{AD}$.

For several decades, neuroanatomical, volumetric, and histological approaches have been the gold standard techniques employed to characterize the OB functionality. However, little attention has been focused specifically on the molecular composition of the OB from the perspective of proteomics [13-15]. We consider that deciphering the progressive proteome-wide alterations that occurs in the $\mathrm{OB}$ derived from $\mathrm{AD}$ cases with different Braak staging, might help develop early diagnosis and identify potential therapeutic targets for $\mathrm{AD}$.

In this study, we used mass-spectrometry based quantitative proteomics as a discovery platform in order to increase our knowledge about the patho-physiological mechanisms that are disturbed during the $\mathrm{AD}$ neurodegeneration in the OB. More than 200 differential proteins between controls and AD-related phenotype were detected, pinpointing specific pathways, protein interaction networks, and potential novel therapeutic targets that are modulated in specific Braak stages. Protein targets mainly involved in dendritic morphogenesis, neuronal injury and axonic distribution were evaluated in a cross-disease study, revealing common and distinct molecular perturbations between different Alzheimerrelated co-pathologies, providing novel candidate proteins for a druggability assessment at the level of the OB.

\section{RESULTS}

In the present study, we have performed immunohistochemical analysis of $\beta$-amyloid and phosphorylated Tau in our OB sample collection derived from $\mathrm{AD}$ subjects (Table 1 ). The detection of $\beta$-amyloid was absent in nonpathological cases and increased along the progress of the disease. The morphology of the deposit was predominantly, in form of mature plaques in advanced stages, while in initial and intermediate stages, predominate the diffuse type of deposit. There was no specific anatomical localization of $A \beta$-protein in the $\mathrm{OB}$ (Figure 1). The detection of phospho-Tau protein was observed in all AD stages in form of neuropil threads and neurofibrillary tangles. The deposit was observed along the different layers of the OB. The intensity of phosphoTau deposit was increased in the anterior olfactory nucleus (AON) of advanced stages of $\mathrm{AD}$ compared to initial stages and control cases where AON phosphoTau staining was negative. In the OB of healthy patients there was only some isolated deposit of phospho-Tau protein (Figure 1). Although some variability in intensity and anatomical localization of protein aggregation were observed between different stages, our data allowed us to confirm the presence of neuropathological proteins in the OB from subjects with different Braak stages, reinforcing the involvement of the $\mathrm{OB}$ in pre-clinical stages of $\mathrm{AD}$.

\section{Large-scale identification of human olfactory bulb proteins by mass spectrometry}

For shotgun proteomics experiments, we have used autopsy specimens of the right $\mathrm{OB}$ structure from $\mathrm{AD}$ cases and controls with no known neurological history (Table 1) with the final goal to obtain a profound insight into the protein content and protein function of the $\mathrm{OB}$ during the progression of $\mathrm{AD}$. To screen the potential differences in $\mathrm{OB}$ protein expression profiles, $\mathrm{OB}$ specimens for each experimental group were separately subjected into isobaric tags (iTRAQ) coupled to 2D nano-liquid chromatography tandem mass spectrometry. Theoretically, pooling samples with a well characterized common pathologic phenotype, reduces the potential for aberrations in inter-sample, or biological variation. This reduction can improve the capacity to identify the most significant and consistent changes between stages. MS/ MS data from OB structure were processed to identify peptides that gave rise to observed spectra, and proteins were inferred based on identified peptides. Using this 
Table 1: Subjects included in the proteomic discovery phase.

\begin{tabular}{|c|c|c|c|c|c|c|c|c|c|c|}
\hline \multirow[t]{2}{*}{ Cases } & \multirow[b]{2}{*}{ age } & \multirow[b]{2}{*}{ sex } & \multirow{2}{*}{$\begin{array}{c}\text { Duration } \\
\text { (years) }\end{array}$} & \multirow{2}{*}{$\begin{array}{c}\text { Brain } \\
\text { weight }(g)\end{array}$} & \multirow{2}{*}{$\begin{array}{c}\text { PMI } \\
\text { (hours) }\end{array}$} & \multirow{2}{*}{$\begin{array}{c}\text { Pathological diagnosis } \\
\text { NIA-AA criteria }\end{array}$} & \multicolumn{2}{|c|}{$I H Q: \beta A$ in $O B$} & \multicolumn{2}{|c|}{$I H Q: T A U$ in $O B$} \\
\hline & & & & & & & $M P$ & $D P$ & Tangles & neurites \\
\hline \multicolumn{11}{|c|}{ Advanced stages } \\
\hline $\mathrm{A} 1$ & 77 & $\mathrm{~F}$ & 16 & 946 & 4 & $\mathrm{AD}(\mathrm{A} 2 \mathrm{~B} 3 \mathrm{C} 3)$ & + & +++ & +++ & +++ \\
\hline $\mathrm{A} 2$ & 70 & $M$ & 4 & 1104 & 2.5 & $A D(A 3 B 3 C 3)$ & ++ & +++ & ++ & +++ \\
\hline A3 & 89 & $M$ & 13 & 1015 & 3 & $A D(A 2 B 3 C 3)$ & + & +++ & +++ & +++ \\
\hline A4 & 86 & $M$ & 8 & 973 & 2.5 & $\mathrm{AD}(\mathrm{A} 3 \mathrm{~B} 3 \mathrm{C} 3)$ & + & - & + & ++ \\
\hline A5 & 93 & $M$ & 3 & 1050 & 2.4 & $A D(A 3 B 3 C 3)$ & + & +++ & +++ & +++ \\
\hline \multicolumn{11}{|c|}{ intermediate stages } \\
\hline M1 & 85 & $M$ & 12 & 1115 & 3.3 & $\mathrm{AD}(\mathrm{A} 2 \mathrm{~B} 2 \mathrm{C} 2)$ & - & + & +++ & +++ \\
\hline M2 & 97 & $\mathrm{~F}$ & 9 & 900 & n.d & $\mathrm{AD}(\mathrm{A} 2 \mathrm{~B} 2 \mathrm{C} 2)$ & n.d & n.d & n.d & n.d \\
\hline M3 & 77 & M & 17 & 1103 & 1.5 & $\mathrm{AD}(\mathrm{A} 2 \mathrm{~B} 2 \mathrm{C} 1)$ & - & - & ++ & ++ \\
\hline M4 & 99 & $\mathrm{~F}$ & n.d & 1002 & 2.3 & $\mathrm{AD}(\mathrm{A} 2 \mathrm{~B} 2 \mathrm{C} 3)$ & n.d & n.d & n.d & n.d \\
\hline M5 & 86 & $\mathrm{~F}$ & 9 & 1000 & 3 & $\mathrm{AD}(\mathrm{A} 2 \mathrm{~B} 2 \mathrm{C} 2)$ & - & + & ++ & ++ \\
\hline \multicolumn{11}{|c|}{ Initial stages } \\
\hline 11 & 88 & $M$ & 1 & 1400 & 3.45 & $\mathrm{AD}(\mathrm{A} 2 \mathrm{~B} 1 \mathrm{C} 2)$ & ++ & + & ++ & ++ \\
\hline 12 & 85 & $\mathrm{~F}$ & 8 & 1130 & 2 & $\mathrm{AD}(\mathrm{A} 2 \mathrm{~B} 1 \mathrm{C} 1)$ & - & - & + & + \\
\hline 13 & 80 & $M$ & 5 & 1090 & 3 & $\mathrm{AD}(\mathrm{A} 2 \mathrm{~B} 1 \mathrm{C} 1)$ & ++ & ++ & ++ & +++ \\
\hline 14 & 75 & $\mathrm{~F}$ & n.d & 1125 & 6 & $A D(A 1 B 1 C 1)$ & - & - & + & + \\
\hline 15 & 72 & $\mathrm{~F}$ & n.d & 810 & 4 & $A D(A 1 B 1 C 1)$ & - & - & + & + \\
\hline \multicolumn{11}{|l|}{ Control } \\
\hline $\mathrm{C} 1$ & 66 & $M$ & & 1233 & 6.3 & No protein deposit+vascular disease & n.d & n.d & n.d & n.d \\
\hline $\mathrm{C} 2$ & 72 & M & & 1407 & 9 & Thal 1 Cerad 1 no tau deposit & - & - & - & + \\
\hline $\mathrm{C} 3$ & 103 & M & & 992 & 3 & No protein deposit+vascular disease & - & - & - & + \\
\hline C4 & 81 & $\mathrm{~F}$ & & 1176 & 3.3 & PART (Braak I)+vascular disase & - & - & - & + \\
\hline C5 & 61 & M & & 1397 & 8 & PART (Braak I) & - & - & + & - \\
\hline
\end{tabular}

The neuropathological assessment was performed according to Thal phases, CERAD score, NIA-AA guidelines and PART criteria [80-83]. $\beta$ A immunopositivity was scored on a 4-tiered scale as: $(-)$ negative, $(+)$ 1-2 isolated $\beta$ A depositions, $(++)$ 3-4 $\beta$ A depositions, and (+++) $>4 \beta$ A depositions. Graduation of phospho-TAU deposit: (-) negative + : low; ++ : intermediate; +++ high. PMI: post-mortem interval; n.d: not determined; MP: Mature plaques; DP: Diffuse plaques (See neuropathological study section).

workflow, we have generated an OB reference proteome map of 4,531 unique proteins identified with at least two peptides with $\geq 95 \%$ confidence (FDR lower than $1 \%$ ). Complete lists of identifications and their corresponding scores are presented in Online Resources 2-3. To extract biological knowledge, the integrated $\mathrm{OB}$ proteome dataset was functionally categorized based on several pathway databases (online Resource 4). Although each bioinformatics platform produced diverse results, they commonly point out that tricarboxylic acid cycle, ubiquitin proteasome pathway and energy-releasing pathways are the general over-represented processes in $\mathrm{OB}$ as might be expected given the high metabolic demands of neurons (Online Resource 4). In order to gain a more detailed description of specific-neuronal pathways detected in the $\mathrm{OB}$, subsequent analyses were performed to explore the proteome distribution across specific reactions using the PANTHER classification system [16]. Some statistically over-represented processes were directly relevant to synaptic vesicle trafficking, integrin signaling pathway, and histamine, dopamine, metabotropic glutamate and muscarinic acetylcholine receptor pathways (Online Resource 1-Figure 2, and Online Resource 4).

\section{OB proteome dynamics during the AD progression}

To further understand the $\mathrm{OB}$ molecular background contributing to the progression of $\mathrm{AD}$, we have performed a differential $\mathrm{OB}$ proteome analysis in order to detect early and stage-dependent molecular events underlying the progression of $\mathrm{AD}$. As expected, we have detected a substantial heterogeneity within the same Braak staging. This may be due to unpredictable confounders such as clinical, environmental, behavioral and agonal factors (i.e medication, substance abuse and health status prior to death) [17]. Among the 4531 identified proteins, 231 proteins tend to be differentially expressed between controls and $\mathrm{AD}$ phenotypes (See Online Resource 1-Figure 3, and Online Resource 5 and 6). In order to gain a more detailed description of the molecular mechanisms involved in the $\mathrm{OB}$ during $\mathrm{AD}$ progression, subsequent analyses were performed to explore the differential olfactory proteome distributions across specific pathways (Online Resource 6). As shown in Figure 2, our results point out a stage-dependent deregulation of specific pathways. Protein clusters involved in DNA repair and biological oxidations were specifically mapped in initial stages while protein groups involved in cell cycle, and signaling by insulin receptor, Rho GTPases, and GPCR 
were exclusively detected in intermediate stages. In advanced stages, specific protein mediators of metabolism of nucleotides and opioid signaling were de-regulated (Online Resource 6). On the other hand, a de-regulation in protein clusters related to axon guidance, and signaling by PDGF, NGF, and EGFR appears throughout intermediate and advanced stages. Interestingly, a cumulative proteome disturbance is detected in energy-integrating and diabetes pathways during the $\mathrm{AD}$ progression at the level of the
OB, suggesting novel experimental pieces of evidence that link diabetes with dementia [18].

To enhance the analytical outcome of proteomic experiments, we have performed proteome-scale interaction networks merging the olfactory proteins that tend to be de-regulated across stages of AD. Using STRING software, a protein interactome map has been constructed for each stage. In initial stages, the heterogeneous nuclear ribonucleoprotein system was
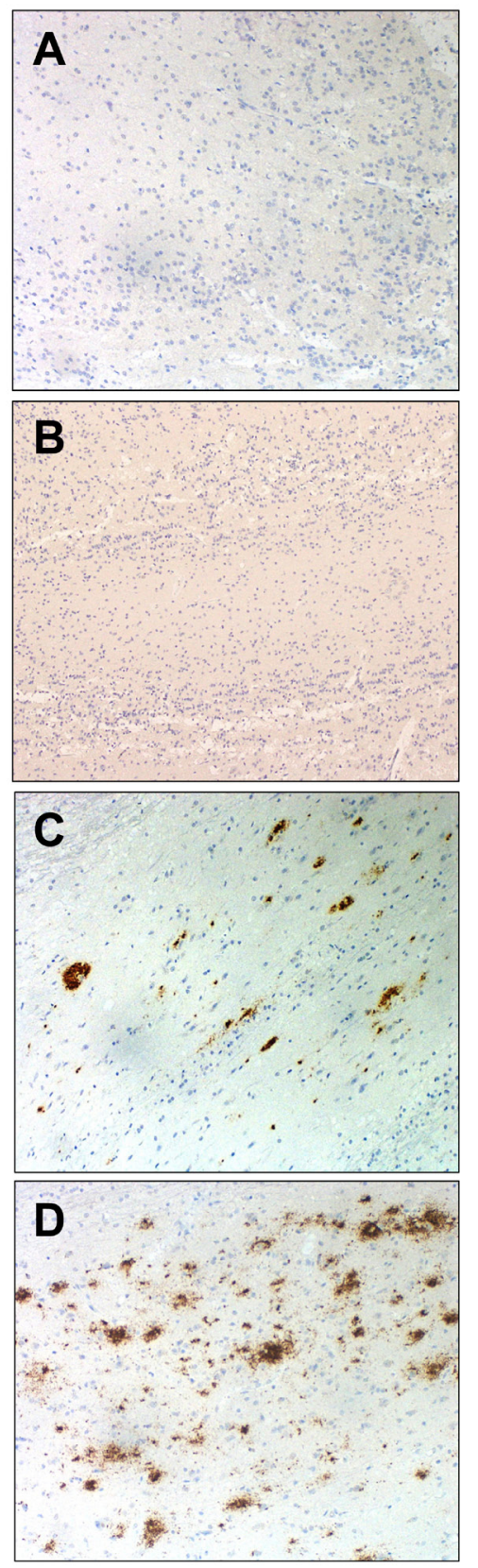
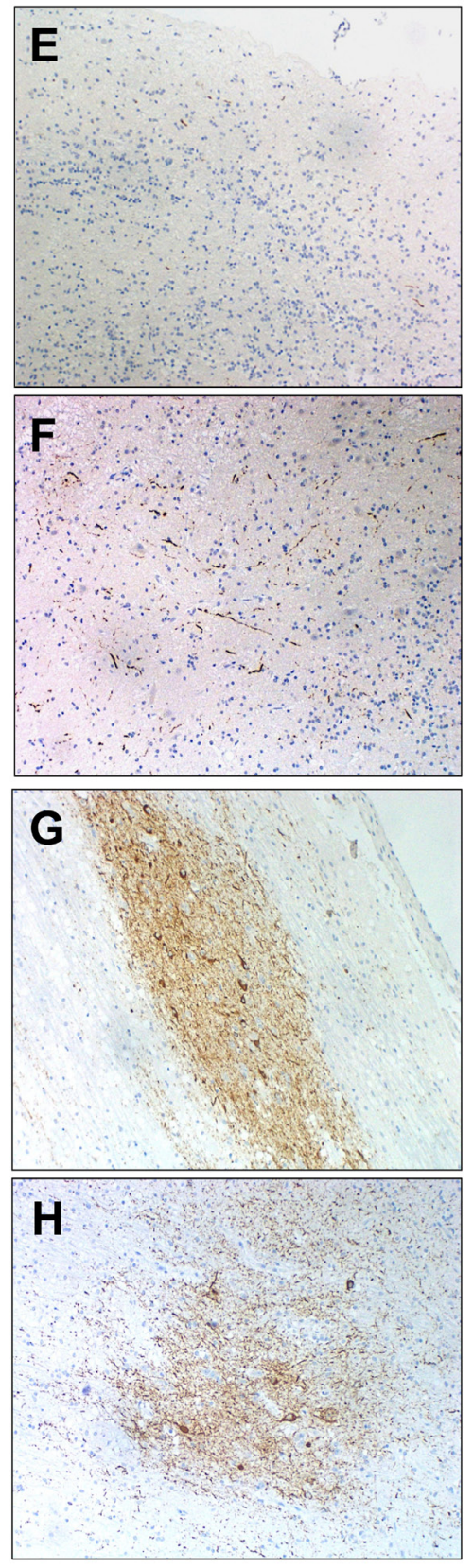

Figure 1: Representative immunohistochemical analysis of Beta-amyloid and p-Tau in OB. Negative Beta-amyloid staining in control A. and initial stage B. moderate staining in intermediate stage C. and abundant deposit in form of diffuse and mature plaques in advanced stage D. Sparse neuropil p-Tau threats in OB glomerular layer in a control case E. p-Tau deposit in external layer of OB (initial stage) F. p-Tau threats and tangles in anterior olfactory nucleus (intermediate stage) G. and abundant deposit of p-Tau in anterior olfactory nucleus (advanced stage) H. (all images are 20X). 


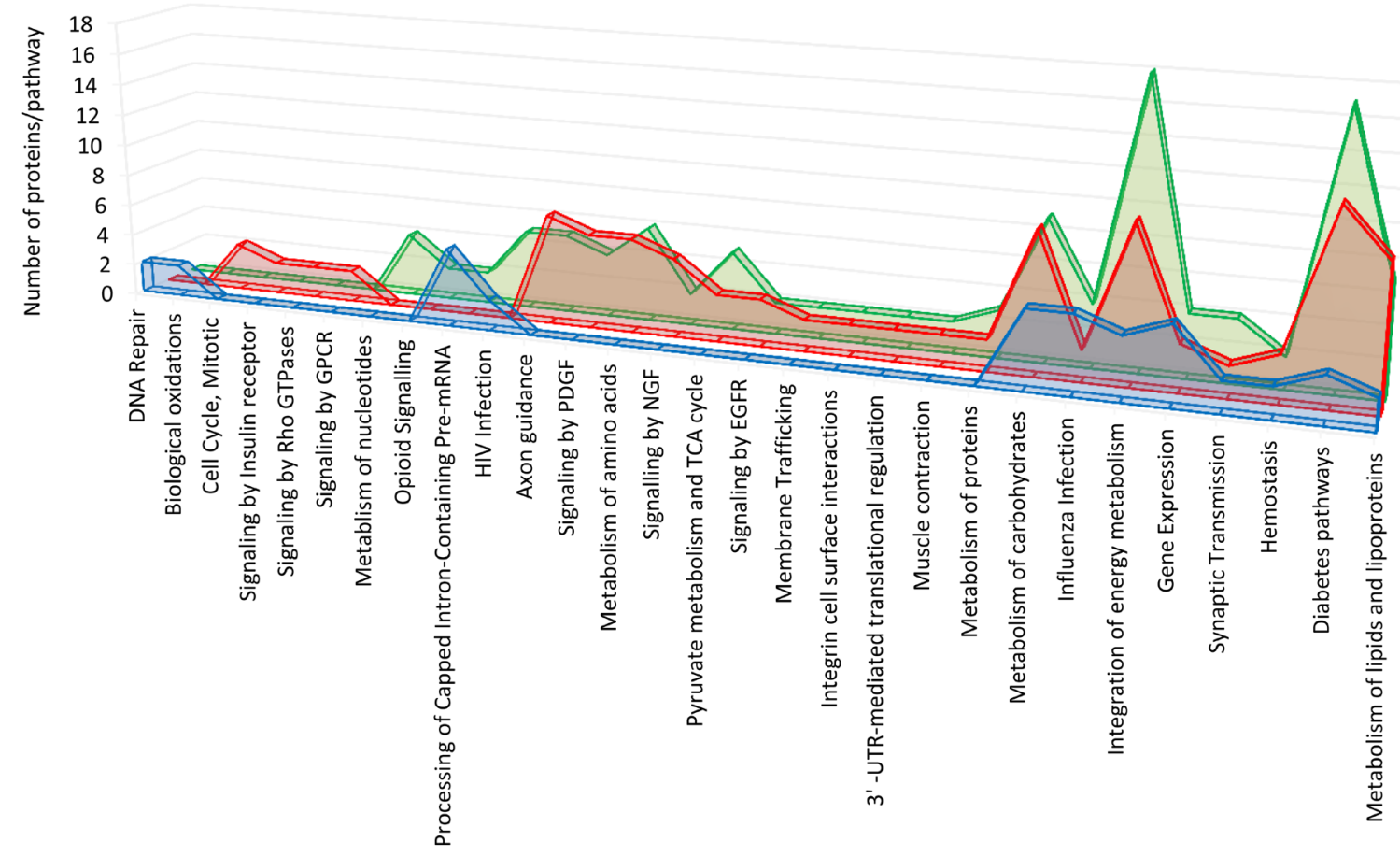

Figure 2: Differential olfactory proteome distributions across specific pathways. Using Reactome database, differential OB proteome across AD stages was mapped into regulatory pathways (See Online Resource 6 for more details).

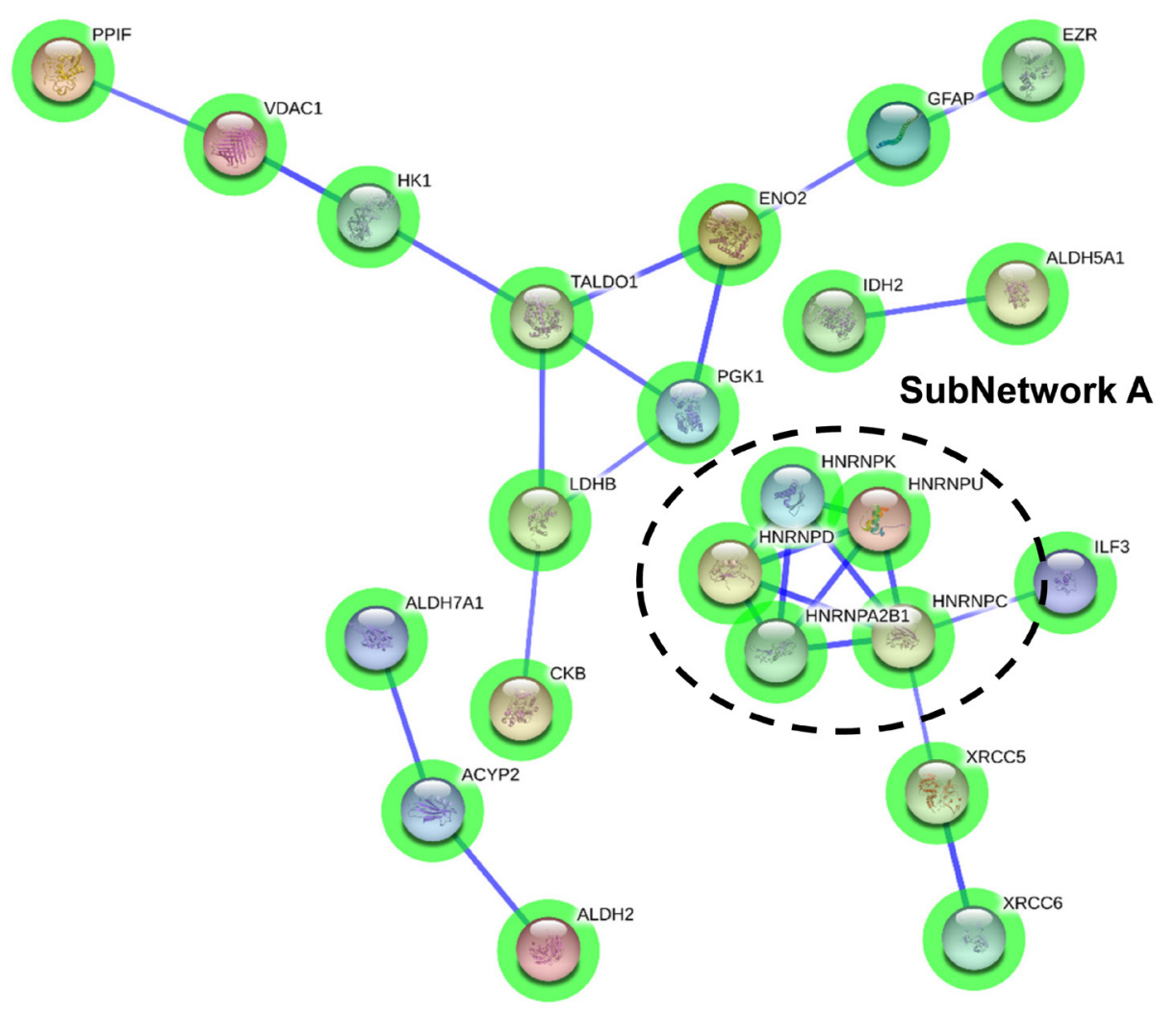

Figure 3: Protein interactome map for differentially expressed proteins in initial stages. STRING analysis of known and predicted protein-protein interactions of gene-products differentially expressed in Braak stages I-II. STRING Version 9.1 and STRINGdb were used. Green circles: down-regulated proteins; red circles: up-regulated proteins. 
compromised in OB (alteration in HNRNPs; subnetwork A) (Figure 3), whereas a deregulation in protein composition of V-type proton ATPase (subnetwork B), collagen (up-regulation of COL6, COL14, COL18; subnetwork C), 14-3-3 complexes (down-regulation of $Y W H A$ proteins; subnetwork D), and a disturbance in neuron-neuron adhesion (LICAM, NCAM1, ALCAM, $N C A N, B C A N$; subnetwork E) appears in intermediate stages (Figure 4). In advanced stages, alterations in mitochondrial complex I (NDUFs), complex II (SDHB), complex III (UQCRC2), and complex V (ATP5) suggest an impaired mitochondrial function (subnetwork $\mathrm{G}$ ) (Figure 5). Moreover, an imbalance in the neurotransmitter cycling ( $A T P 6 V s, A P-1, A P-2$; subnetwork F), and a disturbance in neuron-neuron adhesion and neurite growth (L1CAM, $N C A M 1, A L C A M, N C A N, B C A N$; subnetwork E) also appear in advanced stages (Figure 5).

\section{Proteome-specific changes across AD staging}

A total of $25 \mathrm{OB}$ proteins were significantly differentially expressed proteins in specific stages (Table 2 and Online Resource 1-Figure 4). We compared these results with previous published data on protein differential abundance in AD generated by similar proteomic workflows. Out of 25 proteins, 10 were mentioned as differentially abundant in CSF, cortex, or hippocampus derived from AD subjects [19-24] (Table 2 and Online Resource 5). We investigate whether some differential olfactory proteins are locally modified at the first stages of AD-related pathology when morphological lesions are restricted to the entorhinal and transentorhinal cortices of

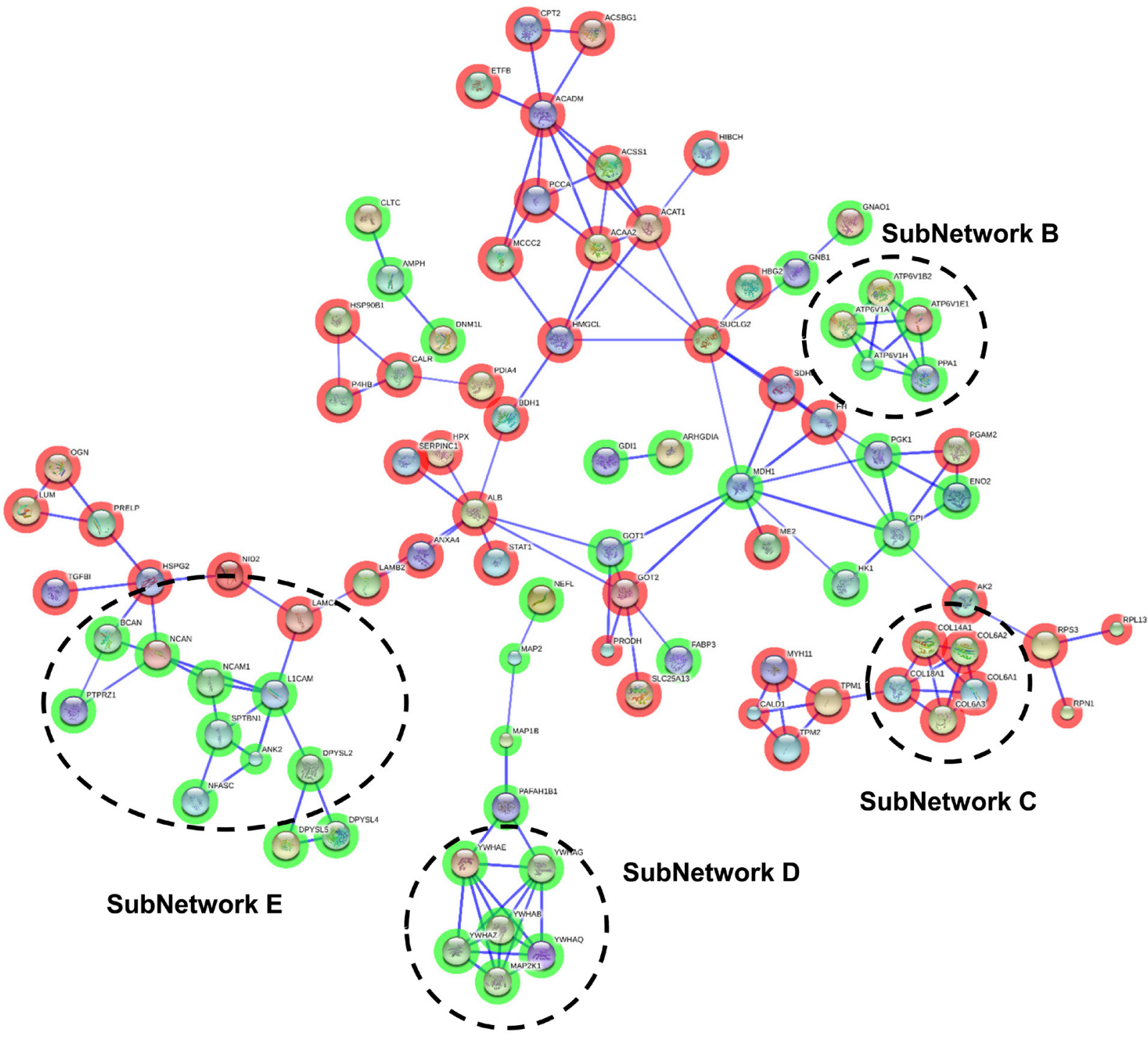

Figure 4: Protein interactome map for differentially expressed proteins in intermediate stages. STRING analysis of known and predicted protein-protein interactions of gene-products differentially expressed in Braak stages III-IV. STRING Version 9.1 and STRINGdb were used. Green circles: down-regulated proteins; red circles: up-regulated proteins. 
neurofibrillary pathology (Braak I-II). For that, Copine-6 (CPNE6), Visinin-like protein 1 (VILIP1), Dipeptidyl aminopeptidase-like protein 6 (DPP6), and RUFY3 protein (RIPX) were further analyzed. To our knowledge, CPNE6, DPP6, and RIPX have not previously been associated with the pathogenesis and progression of AD. However, VILIP1 is a secretable calcium-mediated neuronal injury marker with potential diagnostic utility for early $\mathrm{AD}[25,26]$. Independent of its role as an auxiliary subunit of Kv4-mediated A-type K (+) channels [27], DPP6 participates in the dendritic morphogenesis during the neuronal development in the hippocampus [28]. RIPX is a poorly characterized protein involved in the formation of a single axon by developing neurons [29]. CPNE6 has been described as a protein regulator of axon maturation in the olfactory tract [30]. As shown in Figure 4 and 5, subnetwork $\mathrm{C}$ showed a de-regulation of Collagen VI chains in intermediate/advanced stages. Some studies have demonstrated that collagen VI protects neurons against $\beta$-amyloid toxicity [31]. Using FpClass tool as a data mining-based method for proteome-wide proteinprotein interaction (PPI) predictions (http://dcv.uhnres.

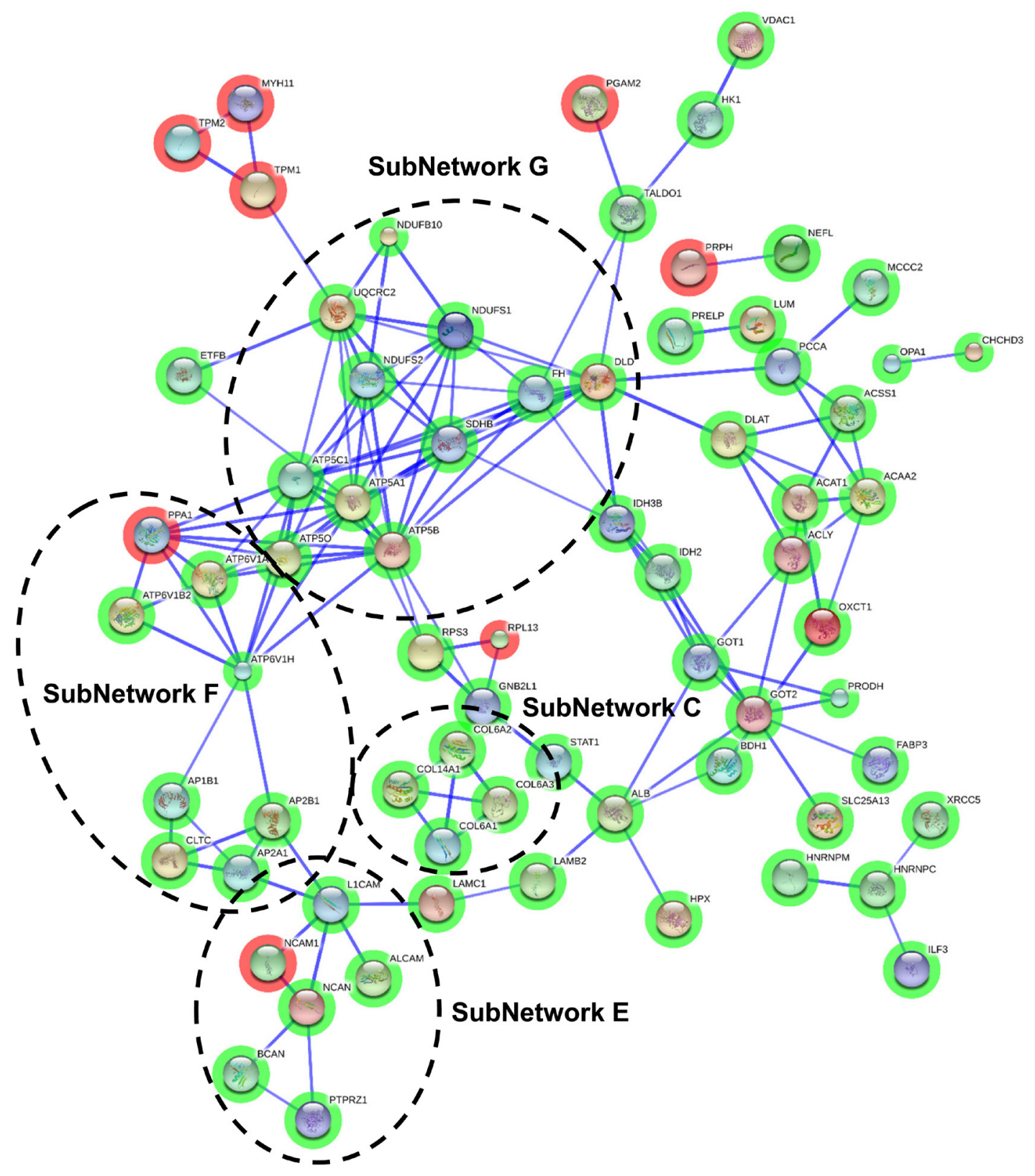

Figure 5: Protein interactome map for differentially expressed proteins in advanced stages. STRING analysis of known and predicted protein-protein interactions of gene-products differentially expressed in Braak stages V-VI. STRING Version 9.1 and STRINGdb were used. Green circles: down-regulated proteins; red circles: up-regulated proteins. 
Table 2: Top proteins significantly differentially expressed in specific AD stages.

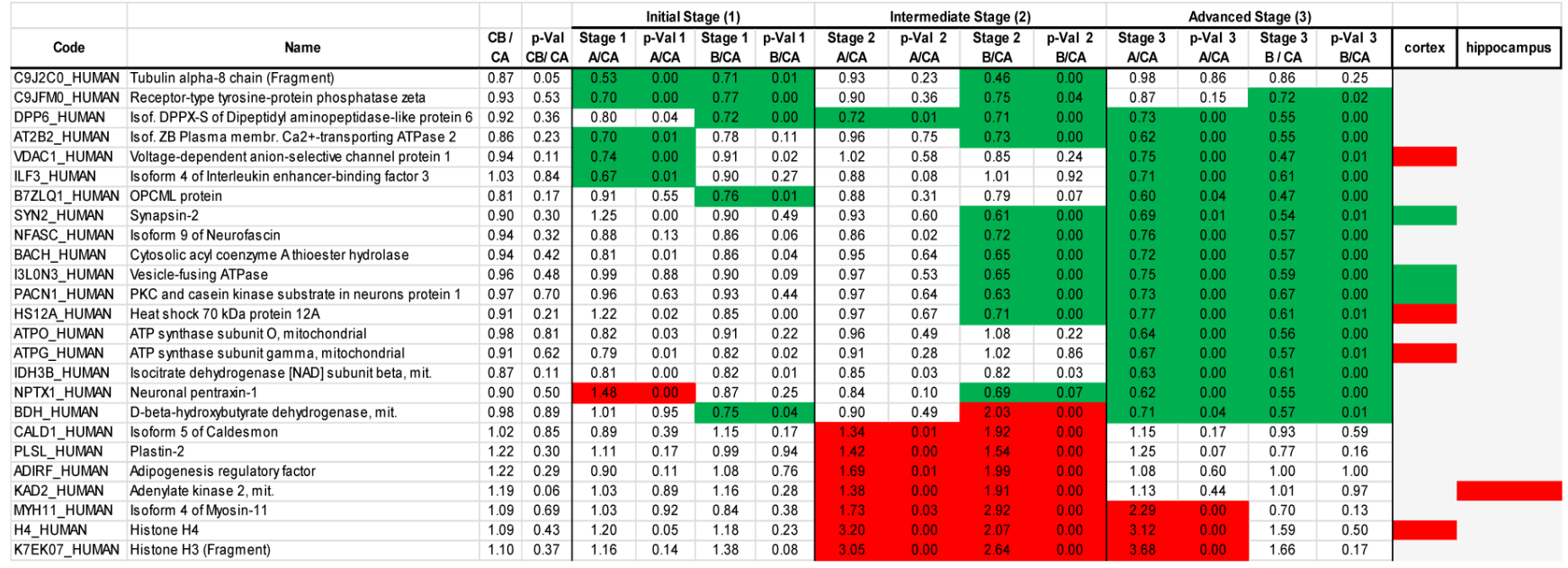

CB, CA (control group A and B), Initial Stage (A and B), Intermediate Stage (A and B), and Advanced Stage (A and B) correspond to the iTRAQ experimental design (see Online Resource 1-figure 1). Ratio corresponds to the protein reporter ion intensity originating from control group B (tag114), Initial stage group A (tag115), Initial stage group B (tag116), Intermediate Stage group A (tag117), Intermediate Stage group B (tag118), Advanced Stage group A (tag119), Advanced Stage group B (Tag121) relative to control group A (tag113). Proteins were considered to show a significant downward (green) or upward (red) trend if their expression ratios were $<0.77$ or $>1.3$ respectively. Proteins mentioned in previous reports as differentially abundant in cortex, or hippocampus derived from AD subjects are indicated.

utoronto.ca/FPCLASS/) [32], we showed that AKAP12 protein (Src-suppressed protein kinase $\mathrm{C}$ substrate) is a putative interactor of Collagen VI chains. This protein increases tau phosphorylation and promotes $\beta$-amyloid toxicity in neuron-like cells [33]. First, we performed immunohistochemical analysis to localize these proteins in the OB region. As shown in Figure 6, RIPX tends to be expressed and preferentially distributed in the glomerular and mitral layers of the OB (Figure 6A). However, CPNE6 is highly expressed by granular and mitral cell layers. In some AD cases, we detected a focal deposit in glomerular structures (Figure 6B). VILIP1 staining is diffuse in glomerular layer presenting focal protein deposit in some areas (Figure 6C). On the other hand, DPP6 is basically detected across neuropil in granular and mitral cell layers, presenting in some cases negative staining in the glomerular layer (Figure 6D). In the case of AKAP12, positive staining was detected at dendritic connections in glomerular layer (Figure 6E). In some AD cases, AKAP12 expression was also detected in endothelial cells (Figure $6 \mathrm{E})$.

Despite the fact that inter-individual variability generally is muted by pooling strategy, validation of individual cases using downstream assays is needed. With the aim to complement and partially validate the iTRAQbased LC-MS/MS analysis, subsequent experiments were performed in order to check the steady-state levels of these proteins in individual cases by Western blotting. In accordance with our proteomic findings, olfactory VILIP1 was transiently up-regulated between early and intermediate stages respect to neurologically intact controls (Figure 7). In order to complement the tendency to DPP6 down-regulation in $\mathrm{AD}$ phenotypes detected by proteomics (Table 2), Western blot analysis were performed across AD stages. As shown in Figure 7, statistically significant differences were detected in olfactory DPP6 protein levels in advanced stages. These data suggest that the dendritic morphology and the number of functional synapses in OB neurons may be compromised in advanced stages of the disease [28]. As shown in Figure 7, RIPX was significantly over-expressed in the OB across early and advanced $\mathrm{AD}$ stages compare to controls, suggesting that RIPX overexpression may enhance the axon length, reducing the percentage of olfactory neurons with multiple axons in AD [29]. As shown in Figure 7, AKAP12 protein expression was significantly increased in early stages of AD compared to controls. However, AKAP12 tend to be down-regulated in intermediate stages. These findings reinforce the idea that transient increase in AKAP12 and collagen VI protein levels may be part of the protective mechanisms against $\beta$-amyloid in olfactory neurons of $\mathrm{AD}$ patients [31,33]. Our proteomic analysis revealed a downregulation of CPNE6 expression in a subset of patients across AD phenotypes (Supp table 5). In contrast, Western blot analysis revealed a tendency to down-regulation in early AD stages but no significant differences between AD phenotypes and controls was clearly detected (Figure 6). The divergence observed between mass spectrometry and Western blot data may be due to a possible biased quantification in our pooling strategy [34]. Overall, a consistent trend was observed with proteomics results for the selected proteins with the exception of CPNE6. 


\section{OB protein expression of CPNE6, VILIP1, DPP6, and RIPX across Alzheimer-related co-pathologies}

In contrast to the common separate investigation of NDs, targeted cross-disease studies comparing shared molecular relationships may give new insights into possible olfactory perturbations common for all or some NDs. In order to detect novel protein mediators shared by different Alzheimer-related co-pathologies at the level of the $\mathrm{OB}$, we have evaluated the $\mathrm{OB}$ protein expression of CPNE6, VILIP1, DPP6, and RIPX by
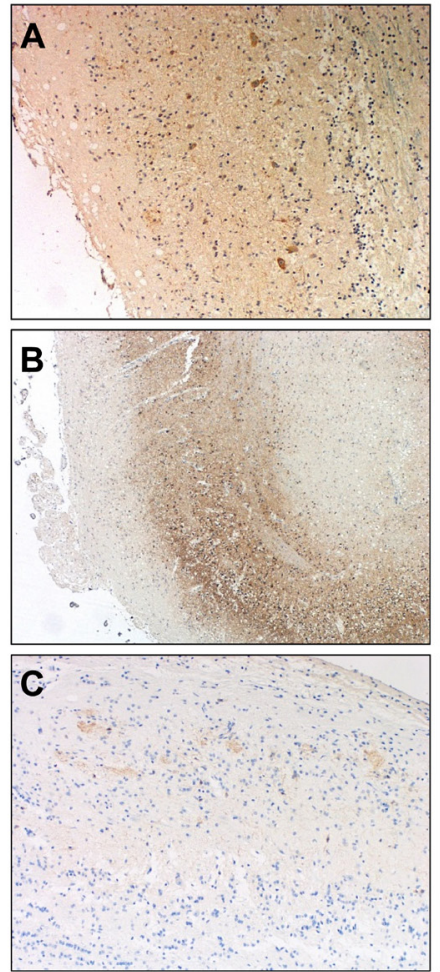

D

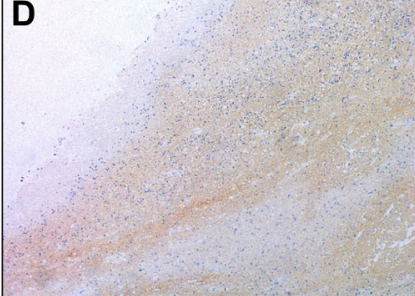

E

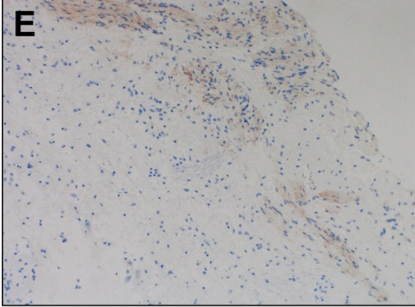

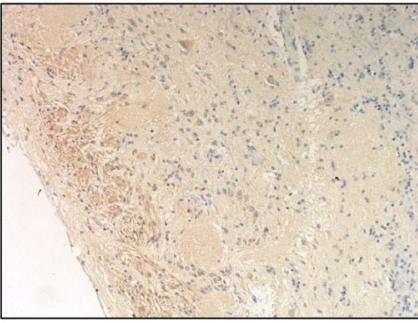
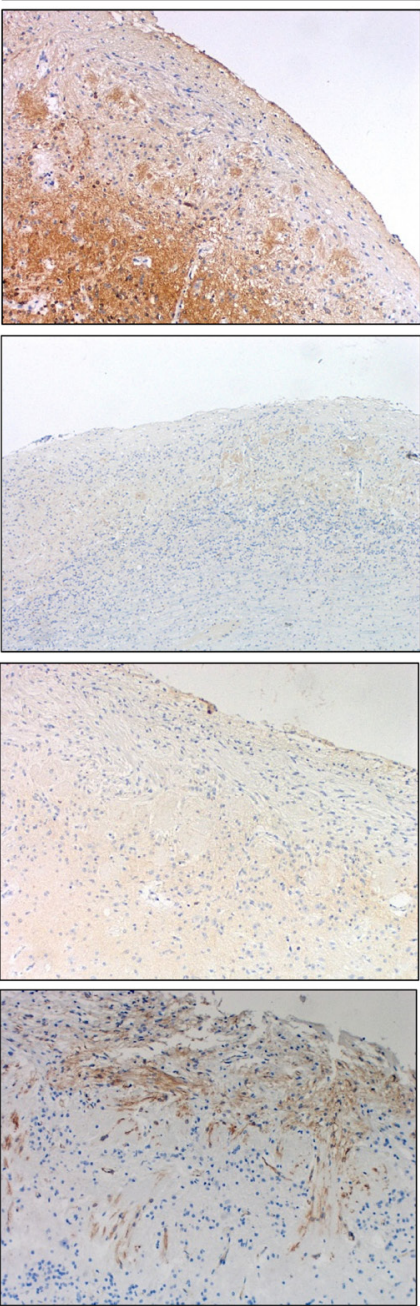
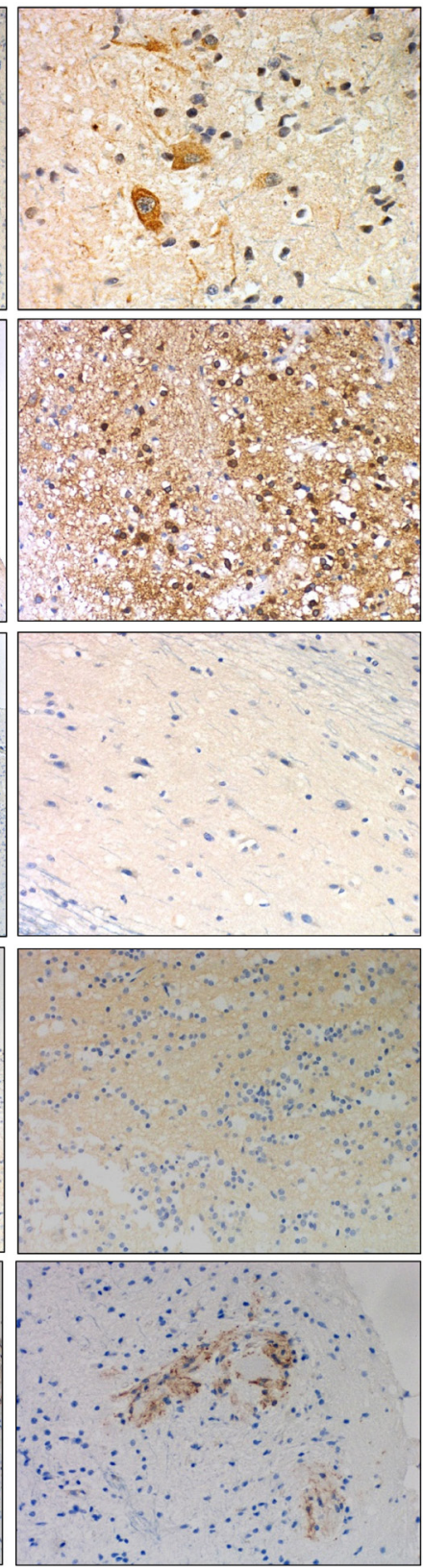

Figure 6: Immunohistochemical localization of AKAP12, RIPX, CPNE6, VILIP1, and DPP6 in human OB. A. Representative immunohistochemical staining pattern of RIPX. Non-pathological OB (left), AD OB (advanced stage) (middle) (4-10X). Detail of RIPX expression in the cytoplasm and axons of mitral cells (40X) (right). B. Representative immunohistochemical staining pattern of CPNE6. Non-pathological OB (left), AD OB (initial stage) (middle) (4-10X). Detail of intense nuclear CPNE6 expression and neuropil deposit in granular cell layer (20X) (right). C. Representative immunohistochemical staining pattern of VILIP1. Non-pathological OB (left), AD OB (Advanced stage) (middle) (10X). Detail of diffuse expression of VILIP1 across neuropil in anterior olfactory nucleus (40X) (right). D. Representative immunohistochemical staining pattern of DPP6. Non-pathological OB (left), AD OB (advanced stages) (middle) (4-20X). Detail of DPP6 expression in granular cells. No evidence of nuclear and cytoplasm deposit (20X) (right). E. Representative immunohistochemical staining pattern of AKAP12. Non-pathological OB (left), AD OB (Advanced stage) (middle) (10X). Detail of AKAP12 expression in dendritic connections in glomerular cell layer (40X). 
Western-blot across several AD-related diseases $(n=$ $41 \mathrm{OB}$ samples). We have included pathologies with common smell impairment like LBD, and FTLD [1], PSP where olfactory loss occurs to a lesser extent or is absent $[1,35,36]$, and mixed dementia (Mix AD VD). Mixed dementia is a condition in which Alzheimer's disease and vascular dementia occur at the same time, and both separate disorders often display olfactory dysfunction $[37,38]$. As shown in Figure 8, expression levels of our protein panel remained unchanged in the OB from PSP subjects respect to controls. Interestingly, although a tendency to DPP6 down-regulation is observed in FTLD and mixed dementia, a significant differential expression is not detected across NDs, suggesting that the alteration of olfactory protein expression of DPP6 tend to be specific for AD (Figure 8). In contrast, RIPX levels are significantly decreased in the OB from LBD, FTLD, and mixed dementia respect to protein levels detected in neurologically intact controls (Figure 8), whereas CPNE6 levels is differentially down-regulated in the OB from LBD and mixed dementia (Figure 8). On the other hand, olfactory VILIP1 expression is significantly up-regulated in FTLD, and mixed dementia. As equivalent olfactory deficits are observed across NDs, our data suggest that specific shared pathways including common pathological substrates are disturbed during the $\mathrm{OB}$ neurodegeneration in some Alzheimer-related co-pathologies.

\section{DISCUSSION}

In view of the involvement of the olfactory dysfunction in $\mathrm{AD}$, we consider that a quantitative knowledge of the olfactory neuroproteome across AD staging may help to understand the early smell impairment that occurs in this disease. The immunohistochemical study of $\beta$-amyloid and phospho-Tau allowed us to confirm the presence of neuropathological proteins in the $\mathrm{OB}$ of
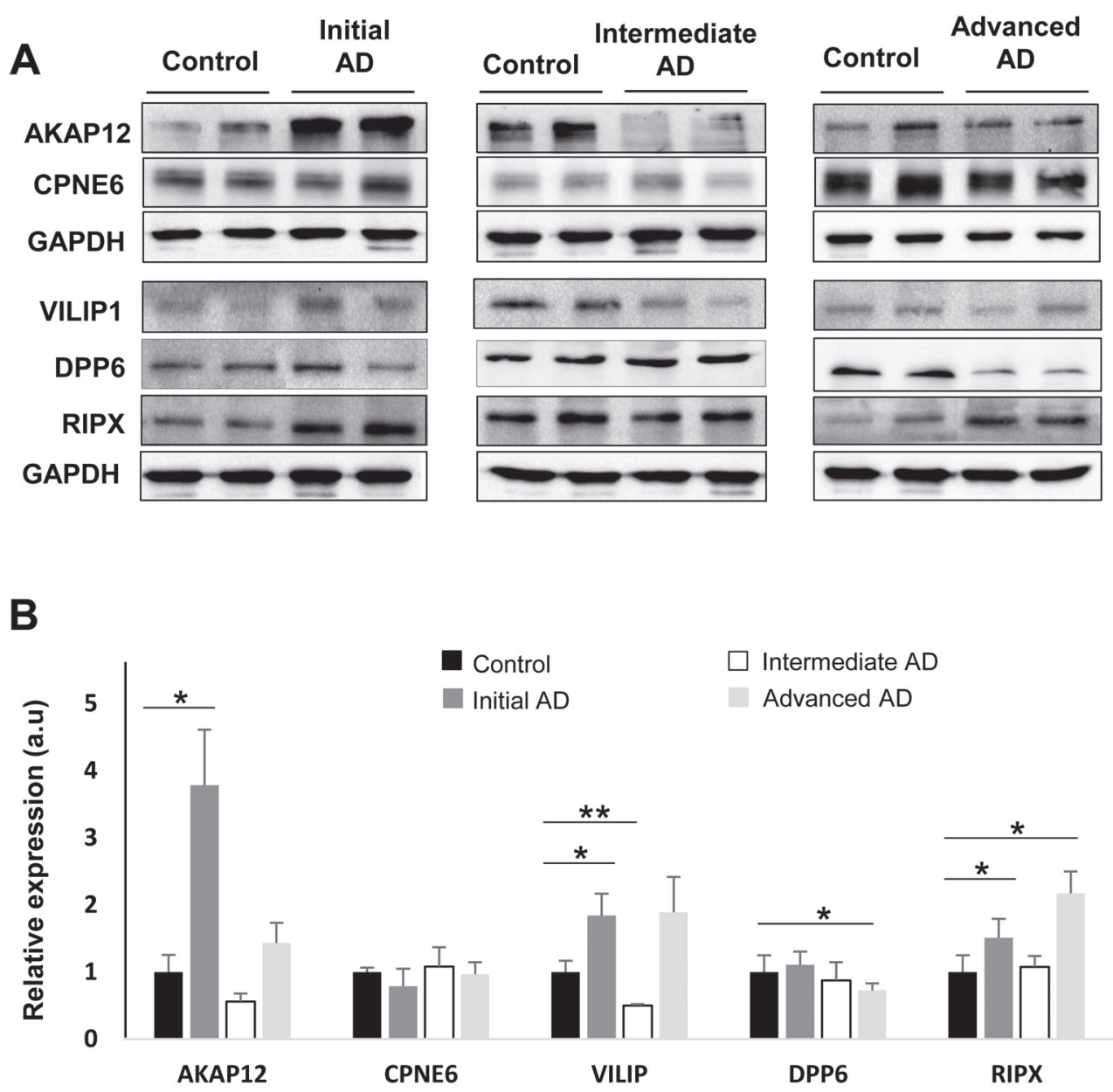

Figure 7: Olfactory bulb protein expression of $A K A P 12, C P N E 6, V I L I P 1, D P P 6$, and $R I P X$ across AD staging. Protein expression were documented by Western blot analyses with antibodies against the respective proteins. Upper panel displays representative Western blot gels ( $n=2$ /experimental group) to detect AKAP12, CPNE6, VILIP1, DPP6, and RIPX protein levels in the OB during the AD progression. Lower panel shows histograms of band densities. Data are presented as mean \pm SEM from five independent OB samples per group. ${ }^{*} P<0.05$ vs control group; ${ }^{*} P<0.01$ vs control group. 
subjects with distinct stages of AD progression, confirming the involvement of $\mathrm{OB}$ in pre-clinical stages of the disease [1]. Although it is widely believed that OB perturbations are responsible for olfactory dysfunction in NDs [1], few studies have examined this area using high throughput molecular technologies. Although transcriptomic analysis have revealed multiple metabolic alterations in the $\mathrm{OB}$ of a rat $\mathrm{AD}$ model [39], the temporal progression of the disease in murine models does not correlate well with human AD [40]. Different neuroproteomic studies have been attempted to discover novel protein mediators associated with AD pathogenesis in brain areas differentially affected by the disease [41-44]. However, to our knowledge, this is the first study to characterize potential AD-associated molecular changes in the $\mathrm{OB}$ using MS-based quantitative proteomics. Basically, the discovery strategy used was based on differential labeling of peptides using isobaric tags prior to their separation and analysis by multidimensional LC coupled to MS [45], revealing new insights into the $\mathrm{OB}$ site-specific proteomic signature during the progression of AD. More than 4,500 proteins have been identified in human $\mathrm{OB}$, being one of the most extensive proteomic characterization of a human brain area [46]. 231 proteins were significantly altered between some $\mathrm{AD}$ phenotypes and neurologically intact controls, where 11 potential biomarkers identified in the OB region (Serum albumin, 14-3-3 protein epsilon, Isoform 3 of Inter-alpha-trypsin inhibitor heavy chain H4, Antithrombin-III, Hemopexin, C4b-binding protein alpha chain, Gamma enolase, Phosphatidylethanolamine binding protein 1, Glial fibrillary acidic protein, Neuronal pentraxin-1, and Neurofascin) (see Online Resource 5) have already been proposed for their potential usefulness in $\mathrm{AD}$ diagnosis $[23,24,47]$. Interestingly, we have detected a down-regulation of specific HSP70 protein in $\mathrm{OB}$ from $\mathrm{AD}$ subjects (see Table 2). Intranasally-
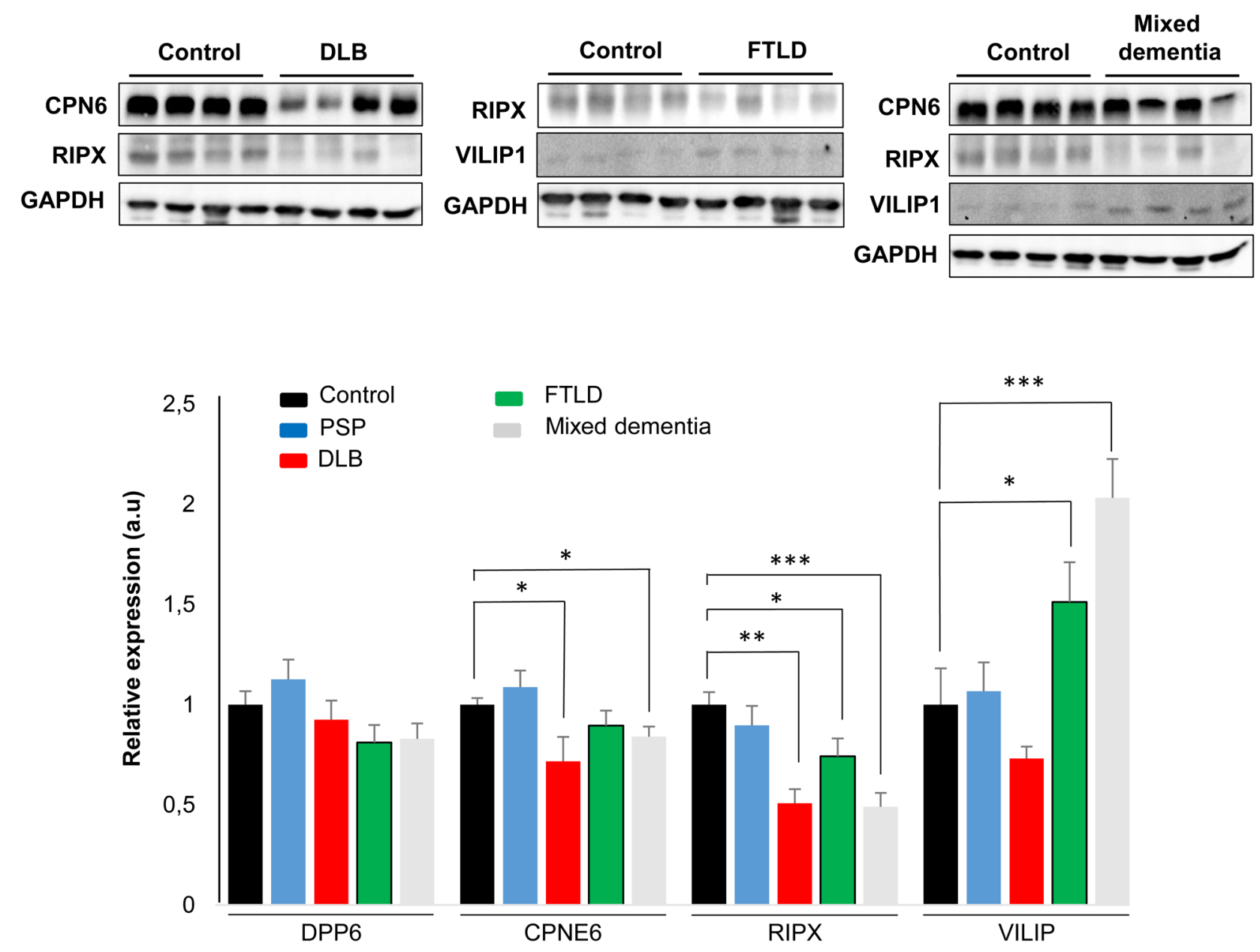

Figure 8: Olfactory expression of CPNE6, VILIP1, DPP6, and RIPX proteins across Alzheimer-related co-pathologies. (Upper panel) Representative Western blot gels ( $\mathrm{n}=4$ /clinical background) to detect CPNE6, VILIP1, and RIPX protein levels in the OB across different NDs. OB DPP6 protein levels remains unchanged across all neurodegenerative syndromes (data not shown). (Lower panel) Histograms of band densities derived from 41 independent OB samples. Data are presented as mean \pm SEM from: Controls $(n=5$ cases), $\operatorname{PSP}(n=10$ cases), LBD ( $n=10$ cases), FTLD ( $n=6$ cases), and mixed dementia (mix AD VD) ( $n=10$ cases). $* P<0.05$ vs control group; ** $P<0.01$ vs control group; $* * * P<0.001$ vs control group. 
administered of recombinant human HSP70 in murine models of $\mathrm{AD}$ has demonstrated dramatic neuroprotective effects [48], suggesting that our experimental workflow is a useful approach to detect and identify practical therapeutic agents for AD treatment. Using data miningbased methods for proteome-scale PPIs predictions [32], we have generated the potential interactome for human APP ( $\beta$-amyloid precursor protein) and Tau proteins (Online Resource 8), detecting some OB differentially expressed proteins during $\mathrm{AD}$ progression as potential APP and/or Tau interactors (Online Resource 1 Figure 5). Although these assumptions should be experimentally validated $[49,50]$, this predictive information may be useful to generate new working hypothesis to clarify the relationship between both neuropathologic substrates in AD.

One of the goals of the present study was to generate extensive data on the functional groups of proteins involved in the neurodegenerative process that occur in the OB during AD pathogenesis. For that, we have undertaken a system biology approach performing different molecular networks and protein profiling analysis $[16,51,52]$ in order to identify biologically relevant pathways from large-scale OB proteome data. From a functional point of view, specific proteomic fingerprints are dynamically modulated in a stage-dependent manner throughout the OB. In initial stages, the heterogeneous nuclear ribonucleoprotein (hnRNP) protein complex was downmodulated (hnRNPA/B between others) (subnetwork A). It has been previously demonstrated that sporadic AD entorhinal cortices present a selective loss of hnRNP $\mathrm{A} / \mathrm{B}$ splicing factors leading to the loss of synapses and dendrites [53]. Interestingly, hnRNP A1 up-regulation induces alternative splicing of the amyloid precursor protein (APP) mRNA, which is followed by reduced $\mathrm{A} \beta$ levels [54], suggesting that a fall of the hnRNP A/B protein levels may inversely exacerbate amyloid pathology in the OB.

In intermediate stages, we have detected an imbalance in the protein composition of V-type proton ATPase (subnetwork B). This multi-subunit protein drives the loading of neurotransmitters into synaptic vesicles [55], indicating that the cycling of neurotransmitters at the olfactory synapse is not totally efficient. Moreover, several Collagen VI chains (subnetwork C) and its potential interactor AKAP12 are de-regulated in intermediate stages, suggesting that modulation of its protein levels may be part of the protective mechanisms against $\beta$-amyloid in olfactory neurons of $\mathrm{AD}$ patients $[31,33]$. 14-3-3 protein family plays a pivotal role in Tau phosphorylation [56], and also in oligomerization and aggregation [57]. Up-regulation of 14-3-3 members have been detected in cortex and choroid plexus of AD patients using proteomic technologies [22, 43]. In our case, olfactory 14-3-3 proteins (subnetwork D) tend to be down-regulated in olfactory neurons in intermediate stages. Albeit the brain areas analyzed are different, the apparent discrepancy between previous proteomic studies and our results may also be due to technological reasons. Previous reports have detected over-expression of 14-33 proteins using 2D-electrophoresis. This approach tend to detect proteoforms by resolving spots at different molecular weight and isoelectric point, hampering the analysis of total expression levels of each 14-3-3 isoform. However, it can be assumed that protein regulation determined by iTRAQ-based approach is more trustworthy regarding the overall expression change of a protein as they are not influenced by any isoform effects. In parallel, a disturbance in a subset of cell adhesion molecules (CAMs) and chondroitin sulfate proteoglycans appeared in intermediate stages, remaining altered in advanced stages (subnetwork E). In general, CAMs participate in neuron-neuron adhesion, and triggers signaling pathways to axon growth [58]. Specifically, L1CAM binds to $\beta$-amyloid reducing histopathological hallmarks of $\mathrm{AD}$ in mice [59], while NCAM1 participates in the maturation of the presynaptic endocytic machinery [60]. Furthermore, chondroitin sulfate proteoglycans such as neurocan (NCAN) and brevican (BCAN) are essential in the maintenance of hippocampal long-term potentiation [61, $62]$. In view of these observations, the down-modulation of CAM-proteoglycan interactome may contribute to the $\beta$-amyloid-related synaptic toxicity impairment in the $\mathrm{OB}$ across intermediate-late phases of AD.

At the end stage of the disease, an interrupted synaptic transmission is largely reflected in the OB. There is a generalized inhibition in protein production of specific V-type proton ATPase subunits, Adaptorrelated proteins (APs), clathrin heavy chain (CLTC) and CAM-proteoglycan complexes (subnetworks $\mathrm{E}$ and F), suggesting an immature form of synaptic vesicle recycling in the OB region [60]. Our proteomescale interaction network analysis further revealed an impaired mitochondrial function, based on the downregulation of subunits of mitochondrial respiratory chain complexes I, II, III and V (subnetwork G). This impairment is a common finding in human AD brains, and also in rodent, and cellular AD models [63, 64], where intracellular $\mathrm{A} \beta$ accumulation leads to a decrease electron transfer efficiency, reduce ATP production, and increase ROS production [65]. Overall, our data point out that functional protein interactomes and specific pathways are dynamically modulated across AD staging in the OB, emphasizing the potential impact of stage-dependent analysis using high-throughput proteome screenings.

Although the implementation of a pooling strategy reduce false-positive rates in proteomic experiments and partially control the clinical and pathophysiological heterogeneity [34], we further applied complementary approaches such as immunohistochemistry and Western blotting to analyze in detail the expression of Visininlike protein 1 (VILIP1), Dipeptidyl aminopeptidase- 
like protein 6 (DPP6), Copine-6 (CPNE6) and RUFY3 protein (RIPX) in the OB. Both VILIP1 and RIPX are up-regulated at the first stages of AD-related pathology, when morphological lesions are restricted to the entorhinal and transentorhinal cortices. Interestingly, both proteins are also maintained in higher levels in advanced stages of AD. VILIP1 is a neuronal calcium sensor which CSF concentration is usually elevated in $\mathrm{AD}$ patients compared to control and LBD subjects [25, 26, 66], and predicts rates of future cognitive decline in early $\mathrm{AD}$ [67]. However, the increment in olfactory VILIP1 levels is transient, falling in $\mathrm{AD}$ subjects with intermediate stages in accordance with previous data obtained from cerebral cortex of AD patients [68]. It has been proposed that the down-regulation of VILIP1 may attenuate neuronal signaling pathways regulating the neuroplasticity process, contributing to cognitive decline in initial stages of $\mathrm{AD}$ [69]. In contrast, OB VILIP1 levels were increased in FTLD and mixed dementia subjects in our cross-disease study. Our data suggest that the complex pattern of upand down-regulation of VILIP1 needs to be studied thoroughly in each pathology in order to decipher the specific signaling routes involved in VILIP1-dependent phosphorylation of Tau [70] and the role of VILIP1 in $\beta$-amyloid-induced calcium overload [71]. Interestingly, calcium sensors also modulate A-type $\mathrm{K}(+)$ channels, controlling responses to excitatory synaptic inputs [72]. DPP6 is an auxiliary subunit of Kv4-mediated A-type $\mathrm{K}(+)$ channels [27, 73], which deficiency induces a decrease in A-type current together with an independent reduction in the number of functional excitatory synapses, affecting the excitatory synaptic function and dendritic branch complexity in murine hippocampus $[28,74]$. Our data also indicate a specific late reduction in the OB levels of DPP6 in AD, suggesting a potential contribution of DPP6 in the aberrant synapse stability at the end stage of AD-pathology. On the other hand, CPNE6 protein levels were only altered in the OB derived from LBD and mixed dementia (mix AD VD) indicating that the axon maturation process may be differentially compromised in the olfactory tract across different NDs [30]. RIPX is a poorly characterized protein involved in cytoskeletal dynamics in growth cones [75] to control the neuronal axon elongation [29]. We have observed a reversed olfactory pattern between mixed dementia (Mix AD VD) respect to the protein profile observed in $\mathrm{AD}$, indicating that RIPX may be considered a common protein mediator that plays specific roles in axon guidance across different neuropathological backgrounds, compromising the regulation of cell polarity and membrane trafficking in olfactory neurons [76]. Transcriptional and translational events may explain this difference observed in RIPX protein levels. A possible explanation is that the vascular damage may induce an increment in the RIPX degradation rate at mRNA and/or proteins levels at the level of the OB in mix AD VD. However, we have to take into account that the activation/inhibition of the transcription factor machinery that regulate the transcription of RUFY3 gene may also be compromised (as a consequence of the vascular damage), leading to a decrease in RUFY3 mRNA and protein levels. However, additional validation studies should be conducted employing large cohorts to verify the protein expression changes observed in our sample set.

Although our study has uncovered many intricacies in $\mathrm{OB}$ protein homeostasis during $\mathrm{AD}$ progression, there are potential limitations of our study that warrant discussion. We have analysed dissected areas that contained multiple cell types [77], thus diluting the proteomic contribution of each specific cell type. Furthermore, the analysis of the OB is not sufficient to investigate the full magnitude of proteome modulation in $\mathrm{AD}$ across the olfactory system. Taking into account that neuropathologic substrates also tend to deposit in olfactory tract and olfactory cortex, additional proteomic studies targeted to these olfactory areas will be necessary to complement the proteomic data specifically derived from the OB.

\section{CONCLUSIONS}

In summary, we have used a differential proteomewide approach revealing stage-dependent molecular alterations in the $\mathrm{OB}$ during the $\mathrm{AD}$ progression. The differential proteomes lie in an imbalance in splicing factors, interrupted cycling of neurotransmitters, alteration in toxic/protective mechanisms of $\beta$-amyloid, mitochondria-mediated neurodegeneration, and a disturbance in neuron-neuron adhesion. Thus, our findings provide basic information for understanding the implication of the $\mathrm{OB}$ in the pathophysiology of $\mathrm{AD}$, identifying protein mediators that may be used as potential therapeutic agents or even explored in biofluids as candidate biomarkers for $\mathrm{AD}$ diagnosis and evolution. In addition, our study revealed changes in the OB levels of specific proteins that had not previously been implied in neurological pathogenesis, suggesting that NDs that have markedly different clinical and pathological features present disruption of shared pathways at the level of the OB.

\section{MATERIALS AND METHODS}

\section{Sample collection}

According to the Spanish Law 14/2007 of Biomedical Research, inform written consent form of the Neurological Tissue Bank of Navarra Health Service was obtained for research purposes from relatives of patients included in this study. Fifteen AD cases were distributed into different groups according to specific consensus 
diagnostic criteria [78-80]: low, intermediate, and high neuropathological changes ( $n=5 /$ group) (Table 1). Five cases from elderly subjects with no history or histological findings of any neurological disease were used as a control group (Table 1). All brains considered in the discovery phase had a post-mortem interval (PMI) lower than 10 hours (Table 1). For specificity analysis, different NDs were considered: Progressive supranuclear palsy (PSP) ( $n$ $=10$ cases; $5 \mathrm{~F} / 5 \mathrm{M}$; median age: 74 years), Lewy body disease (LBD) $(n=10$ cases; $6 \mathrm{~F} / 4 \mathrm{M}$; median age: 80 years), frontotemporal lobar degeneration (FTLD) ( $n=6$; $3 \mathrm{~F} / 3 \mathrm{M}$; median age: 81 years), mixed dementia (mix AD VD) ( $n=10$ cases; $5 \mathrm{~F} / 5 \mathrm{M}$; median age: 85 years), and controls ( $n=5 ; 2 \mathrm{~F} / 3 \mathrm{M}$; median age: 79 years). $80 \%$ of the OB samples included in this phase had a PMI lower than 10 hours (See Online Resource 7).

\section{Neuropathological study}

One hemisphere (usually the left) with the corresponding OB was fixed in $10 \%$ formalin for morphological studies. After fixation (21-25 days), representative brain areas from cortical and subcortical areas, brainstem, cerebellum and spinal cord were taken and embedded in paraffin in order to make a neuropathological diagnosis. Neuropathological assessment was performed according to standardized neuropathological scoring/grading systems, including Thal phases of Beta-amyloid deposition, Braak staging of neurofibrillary lesions, Consortium to Established a Registry for Alzheimer's Disease, National Institute on Aging-Alzheimer's Association (NIA-AA) guidelines, primary age-related tauopathy (PART) criteria, McKeith criteria for Lewy body disease, Mackenzie criteria for FTLD pathology, NINDS-AIREN criteria for vascular dementia, and NINDS criteria for PSP [78-87]. OB betaamyloid and Phos-Tau immunostaining were exclusively performed in $\mathrm{AD}$ and control cases. The OBs were embedded in paraffin and 4- $\mu$ m-thick sagittal sections were stained with hematoxylin-eosin and $3-\mu \mathrm{m}$-thick sagittal sections were processed for immunohistochemical analysis. Formalin-fixed sections (3-5 $\mu$ m-thick) were mounted on slides and deparaffinized. After conducting a routine antigen retrieval protocol, tissue sections were immunohistochemically labelled overnight with a mouse monoclonal antibody anti-human PHF-TAU (clone AT8, Innogenetics) and a mouse monoclonal ( $\mathrm{S} 6 \mathrm{~F} / 3 \mathrm{D})$ anti Beta-amyloid (Leica). The reaction product was visualized using an automated slide immunostainer (Leica Bond Max) with Bond Polymer Refine Detection (Leica Biosystems Newcastle Ltd). Analysis of OB for specific protein deposits aggregates was carried out in a light microscope (Olympus BX51) blinded to pathological diagnosis. A semiquantitative assessment was performed according to Kovacs T et al. [88]. We consider compact deposit with central core as mature plaques and granular or fibrillar deposit as diffuse plaques. Moreover, $A \beta$ immunopositivity was scored on a 4-tiered scale as: (-) negative, (+) 1-2 isolated $\mathrm{A} \beta$ depositions, (++) 3-4 $\mathrm{A} \beta$ depositions, and $(+++)>4 \mathrm{~A} \beta$ depositions. We also described different patterns of deposits and its intensity for Phos-Tau immunostaining : neurofibrillary tangles, neuropil threads and neuritic plaques (n.d: not determined; +: low; ++: intermediate; +++ high) (see Table 1). All preparations were examined by two independent pathologists.

\section{Materials}

The following reagents and materials were used: anti-GAPDH (Calbiochem), anti-DPP6 (Sigma), antiAKAP12, anti-CPNE6, anti-RIPX, anti-VILIP1 (Thermo Scientific). Electrophoresis reagents were purchased from Biorad and trypsin from Promega.

\section{Immunoblotting analysis}

Equal amounts of protein $(15 \mu \mathrm{g})$ were resolved in $12.5 \%$ SDS-PAGE gels. OB proteins derived from control, AD, PSP, LBD, FTLD, and mix AD VD were electrophoretically transferred onto nitrocellulose membranes for $45 \mathrm{~min}$ at $120 \mathrm{~V}$. Membranes were probed with primary antibodies at 1:1000 dilution in 5\% nonfat milk. After incubation with the appropriate horseradish peroxidase-conjugated secondary antibody (1:5000), the immunoreactivity was visualized by enhanced chemiluminescence (Perkin Elmer). Equal loading of the gels was assessed by Ponceau staining and hybridization with a GAPDH specific antibody (Calbiochem). Results are expressed as an n-fold increase over the values of the control group in densitometric arbitrary units.

\section{Immunohistochemistry}

For the immunohistochemical study, formalinfixed sections (3-5 mm-thick) were mounted on slides and deparaffinized. Tissue sections were labelled with the following primary antibodies: anti-DPP6 (dilution 1/200), anti-AKAP12 (dilution 1/2500), anti-CPNE6 (dilution 1/500), anti-RIPX (dilution 1/500), anti-VILIP1 (dilution $1 / 100$ ). The reaction product was visualized using an automated slide immunostainer (Leica Bond Max) with Bond Polymer Refine Detection (Leica Biosystems Newcastle Ltd).

\section{Sample preparation for proteomic analysis}

OB specimens derived from control and $\mathrm{AD}$ cases (Table 1) were homogenized in lysis buffer containing 7 $\mathrm{M}$ urea, $2 \mathrm{M}$ thiourea, 4\% (w/v) CHAPS, $50 \mathrm{mM}$ DTT. 
The homogenates were spinned down at $100.000 \mathrm{x} \mathrm{g}$ for $1 \mathrm{~h}$ at $15^{\circ} \mathrm{C}$. Protein concentration was measured in the supernatants with the Bradford assay kit (Biorad). Prior to proteomic analysis, the individual OB samples were grouped into 8 independent pools containing $\sim 200 \mu \mathrm{g}$ of protein from 2-3 individual samples each one (see experimental design in Online Resource 1-Figure 1).

\section{Protein digestion and peptide iTRAQ labeling}

A shotgun comparative proteomic analysis of $\mathrm{OB}$ proteomes using iTRAQ (isobaric Tags for Relative and Absolute Quantitation) was performed [45]. Protein extracts were precipitated with methanol/choloroform, and pellets dissolved in $7 \mathrm{M}$ urea, $2 \mathrm{M}$ thiourea, 4\% (v/v) CHAPS. Protein quantitation was performed with the Bradford assay kit (Bio-Rad). iTRAQ labeling of each pooled sample was performed according to the manufacturer's protocol (ABSciex). Briefly, equal amounts of $\mathrm{OB}$ proteins $(80 \mu \mathrm{g})$ from each pool were reduced with $50 \mathrm{mM}$ tris (2-carboxyethyl) phosphine (TCEP) at $60{ }^{\circ} \mathrm{C}$ for $1 \mathrm{~h}$. Cysteine residues were alkylated with $200 \mathrm{mM}$ methylmethanethiosulfonate (MMTS) at room temperature for $15 \mathrm{~min}$. Protein enzymatic cleavage was carried out with trypsin (Promega; 1:20, w/w) at 37 ${ }^{\circ} \mathrm{C}$ for $16 \mathrm{~h}$. Each tryptic digest was labelled according to the manufacturer's instructions with one isobaric amine-reactive tags as follows (see Online Resource 1-Figure 1): Tag113, control group A; Tag114, control group B; Tag115, Braak stage I-II group A; Tag116, Braak stage I-II group B; Tag117, Braak stage III-IV group A; Tag118, Braak stage III-IV group B; Tag119, Braak stage V-VI group A; Tag121, Braak stage V-VI group B. After $1 \mathrm{~h}$ incubation, each set of labelled samples were independently pooled and evaporated until $<40 \mu \mathrm{l}$ in a vacuum centrifuge.

\section{Liquid chromatography (LC)}

To increase the proteome coverage, the peptide pool was injected to an Ettan LC system with a X-Terra RP18 precolumn $(2.1 \times 20 \mathrm{~mm})$ and a high $\mathrm{pH}$ stable X-Terra

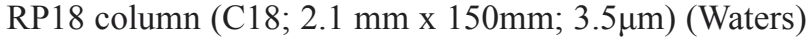
at a flow rate of $40 \mu \mathrm{l} / \mathrm{min}$. Peptides were eluted with a mobile phase B of 5-65\% linear gradient over 35 min (A, $5 \mathrm{mM}$ ammonium bicarbonate in water at $\mathrm{pH} 9.8 ; \mathrm{B}, 5$ $\mathrm{mM}$ ammonium bicarbonate in acetonitrile at $\mathrm{pH} 9.8$ ). 14 fractions were collected, evaporated under vacuum and reconstituted into $20 \mu \mathrm{l}$ of $2 \%$ acetonitrile, $0.1 \%$ formic acid, 98\% MilliQ- $\mathrm{H}_{2} 0$ prior to mass spectrometric analysis.

\section{Mass spectrometry analysis}

Peptides mixtures were separated by reverse phase chromatography using an Eksigent nanoLC ultra 2D pump fitted with a $75 \mu \mathrm{m}$ ID column (Eksigent 0.075 x 150). Samples were first loaded for desalting and concentration into a $0.5 \mathrm{~cm}$ length $300 \mu \mathrm{m}$ ID precolumn packed with the same chemistry as the separating column. Mobile phases were $100 \%$ water $0.1 \%$ formic acid (FA) (buffer A) and $100 \%$ Acetonitrile $0.1 \%$ FA (buffer B). Column gradient was developed in a 70 min two step gradient from $2 \%$ B to $30 \% \mathrm{~B}$ in $60 \mathrm{~min}$ and $30 \% \mathrm{~B}$ to $40 \% \mathrm{~B}$ in $10 \mathrm{~min}$. Column was equilibrated in $95 \% \mathrm{~B}$ for $5 \mathrm{~min}$ and $2 \% \mathrm{~B}$ for $15 \mathrm{~min}$. During all process, precolumn was in line with column and flow maintained all along the gradient at $300 \mathrm{nl} / \mathrm{min}$. Eluting peptides from the column were analyzed using an AB Sciex 5600 Triple-TOF system. Information data acquisition was acquired upon a survey scan performed in a mass range from $350 \mathrm{~m} / \mathrm{z}$ up to $1250 \mathrm{~m} / \mathrm{z}$ in a scan time of $250 \mathrm{~ms}$. Top 25 peaks were selected for fragmentation. Minimum accumulation time for MS/MS was set to 75 $\mathrm{ms}$ giving a total cycle time of $2.1 \mathrm{~s}$. Product ions were scanned in a mass range from $100 \mathrm{~m} / \mathrm{z}$ up to $1500 \mathrm{~m} / \mathrm{z}$ and excluded for further fragmentation during $15 \mathrm{~s}$.

\section{Data analysis}

After MS/MS analysis, data files were processed using ProteinPilot ${ }^{\mathrm{TM}} 4.5$ software from AB Sciex which uses the algorithm Paragon ${ }^{\mathrm{TM}}$ (v.4.0.0.0) [89] for database search and Progroup ${ }^{\mathrm{TM}}$ for data grouping and searched against Uniprot Human database. The search parameters allowed for cysteine modification by MMTS and biological modifications programmed in the algorithm (i.e. phosphorylations, amidations, semitryptic fragments, etc.). Reporter ion intensities were bias corrected for the overlapping isotope contributions from the iTRAQ tags according to the certificate of analysis provided by the reagent manufacturer (ABsciex). The peptide and protein selection criteria for relative quantitation were performed as previously described [90]. Several quantitative estimates provided for each protein by ProteinPilot were utilized: the fold change ratios of differential expression between labelled protein extracts; the p-value, representing the probability that the observed ratio is different than 1 by chance. A decoy database search strategy was also used to estimate the false discovery rate (FDR), defined as the percentage of decoy proteins identified against the total protein identification. The FDR was calculated by searching the spectra against the decoy database generated from the target database using a non-lineal fitting method [91] and displayed results were those reporting a 1\% Global FDR or better. The results were then exported into Excel for manual data interpretation. Although relative quantification and statistical analysis were provided by the 
ProteinPilot software, an additional 1.3-fold change cutoff for all iTRAQ ratios (ratio $\leq 0.77$ or $\geq 1.3$ ) was selected to classify proteins as up- or down-regulated. Proteins with iTRAQ ratios below the low range $(0.77)$ were considered to be underexpressed, whereas those above the high range (1.3) were considered to be overexpressed.

\section{Bioinformatic analysis}

The proteomic information was analyzed using bioinformatic tools including DAVID (Database for Annotation, Visualization and Integrated Discovery) Bioinformatics Resources (v6.7), Panther, and Reactome tools [52, 92]. These programs detect and infer differentially activated/deactivated pathways as a result of AD phenotypes. The identification of specifically dysregulated regulatory/metabolic networks during the AD progression was analysed by STRING (Search Tool for the Retrieval of Interacting Genes) software (v.9.1) (http:// stringdb.org/) [51]. This database includes interactions from published literature describing experimentally studied interactions, as well as those from genome analysis using several well-established methods based on domain fusion, phylogenetic profiling and gene neighbourhood concepts. A higher score was assigned when an association is supported by several types of evidence. To minimize false positives as well as false negatives, all interactions tagged as "low-confidence" $(<0.4)$ in STRING database have been eliminated from this study.

\section{Abbreviations}

AD: Alzheimer's disease

AKAP12: A kinase anchor protein 12

DPP6: Dipeptidyl aminopeptidase-like protein 6

LC: Liquid chromatography

MS: Mass spectrometry

PPI: Protein-protein interaction

RIPX: RUFY3 protein

VILIP1: Visinin-like protein 1

PSP: Progressive supranuclear palsy

LBD: Lewy body disease

FTLD: Frontotemporal lobar degeneration

VD: vascular dementia

\section{ACKNOWLEDGMENTS}

We thank the Neurological Tissue Bank of Navarra Health Service for providing us the OB specimens. The authors appreciate the help from Dr. Federico GarciaBragado from Pathology Department, Hospital Virgen del Camino (Pamplona, Spain). Navarrabiomed Proteomics Unit is a member of ProteoRed, PRB2-ISCIII, and is supported by grant PT13/0001, of the PE I+D+I 20132016 funded by ISCIII and FEDER.

\section{CONFLICTSOF INTEREST}

The authors declare that they have no conflict of interest.

\section{FUNDINGS}

This work was funded by grants from the Spanish Ministry of Economy and Competitiveness (Ref. SAF2014-59340-R) and Caja Navarra Foundation (Ref. 70027) to E.S. XMM obtained a Technology Development Fellowship from Government of Navarra. EPV is financed by the Spanish Ministry of Economy and Competitiveness.

\section{REFERENCES}

1. Attems J, Walker L and Jellinger KA. Olfactory bulb involvement in neurodegenerative diseases. Acta Neuropathol. 2014; 127:459-475.

2. Attems $J$ and Jellinger KA. Olfactory tau pathology in Alzheimer disease and mild cognitive impairment. Clin Neuropathol. 2006; 25:265-271.

3. Doty RL. The olfactory vector hypothesis of neurodegenerative disease: is it viable? Ann Neurol. 2008; 63:7-15.

4. Mundinano IC, Caballero MC, Ordonez C, Hernandez M, DiCaudo C, Marcilla I, Erro ME, Tunon MT and Luquin MR. Increased dopaminergic cells and protein aggregates in the olfactory bulb of patients with neurodegenerative disorders. Acta Neuropathol. 122:61-74.

5. Jellinger KA. Olfactory bulb alpha-synucleinopathy has high specificity and sensitivity for Lewy body disorders. Acta Neuropathol. 2009; 117:215-216; author reply 217218.

6. Mundinano IC, Hernandez M, Dicaudo C, Ordonez C, Marcilla I, Tunon MT and Luquin MR. Reduced cholinergic olfactory centrifugal inputs in patients with neurodegenerative disorders and MPTP-treated monkeys. Acta Neuropathol. 126:411-425.

7. Bahar-Fuchs A, Chetelat G, Villemagne VL, Moss S, Pike $\mathrm{K}$, Masters CL, Rowe C and Savage G. Olfactory deficits and amyloid-beta burden in Alzheimer's disease, mild cognitive impairment, and healthy aging: a PiB PET study. J Alzheimers Dis. 2010; 22:1081-1087.

8. Kovacs T, Cairns NJ and Lantos PL. Olfactory centres in Alzheimer's disease: olfactory bulb is involved in early Braak's stages. Neuroreport. 2001; 12:285-288.

9. Arnold SE, Lee EB, Moberg PJ, Stutzbach L, Kazi H, Han LY, Lee VM and Trojanowski JQ. Olfactory epithelium amyloid-beta and paired helical filament-tau pathology in Alzheimer disease. Ann Neurol. 2010; 67:462-469.

10. Talamo BR, Rudel R, Kosik KS, Lee VM, Neff S, Adelman L and Kauer JS. Pathological changes in olfactory neurons in patients with Alzheimer's disease. Nature. 1989; 
337:736-739.

11. Thomann PA, Dos Santos V, Seidl U, Toro P, Essig M and Schroder J. MRI-derived atrophy of the olfactory bulb and tract in mild cognitive impairment and Alzheimer's disease. J Alzheimers Dis. 2009; 17:213-221.

12. Forster S, Vaitl A, Teipel SJ, Yakushev I, Mustafa M, la Fougere C, Rominger A, Cumming P, Bartenstein P, Hampel H, Hummel T, Buerger K, Hundt W and Steinbach S. Functional representation of olfactory impairment in early Alzheimer's disease. J Alzheimers Dis. 2010; 22:581591.

13. Fernandez-Irigoyen J, Corrales FJ and Santamaria E. Proteomic atlas of the human olfactory bulb. J Proteomics. 2012; 75:4005-4016.

14. Maurya DK, Sundaram CS and Bhargava P. Proteome profile of the mature rat olfactory bulb. Proteomics. 2009; 9:2593-2599.

15. Wille M, Schumann A, Kreutzer M, Glocker MO, Wree A, Mutzbauer G and Schmitt O. The proteome profiles of the olfactory bulb of juvenile, adult and aged rats - an ontogenetic study. Proteome Sci. 2015; 13:8.

16. Mi H, Muruganujan A, Casagrande JT and Thomas PD. Large-scale gene function analysis with the PANTHER classification system. Nat Protoc. 2013; 8:1551-1566.

17. Leite RE and Grinberg LT. Closing the gap between brain banks and proteomics to advance the study of neurodegenerative diseases. Proteomics Clin Appl. 2015; 9:832-7.

18. Verdile G, Fuller SJ and Martins RN. The role of type 2 diabetes in neurodegeneration. Neurobiol Dis. doi: 10.1016/j.nbd.2015.04.008. [Epub ahead of print].

19. Musunuri $\mathrm{S}$, Wetterhall $\mathrm{M}$, Ingelsson $\mathrm{M}$, Lannfelt $\mathrm{L}$, Artemenko K, Bergquist J, Kultima K and Shevchenko G. Quantification of the brain proteome in Alzheimer's disease using multiplexed mass spectrometry. J Proteome Res. 2014; 13:2056-2068.

20. Zahid S, Oellerich M, Asif AR and Ahmed N. Differential expression of proteins in brain regions of Alzheimer's disease patients. Neurochem Res. 2014; 39:208-215.

21. Manavalan A, Mishra M, Feng L, Sze SK, Akatsu H and Heese K. Brain site-specific proteome changes in agingrelated dementia. Exp Mol Med. 2013; 45:e39.

22. Andreev VP, Petyuk VA, Brewer HM, Karpievitch YV, Xie F, Clarke J, Camp D, Smith RD, Lieberman AP, Albin RL, Nawaz Z, El Hokayem J and Myers AJ. Label-free quantitative LC-MS proteomics of Alzheimer's disease and normally aged human brains. J Proteome Res. 2012; 11:3053-3067.

23. Zhang J, Goodlett DR, Quinn JF, Peskind E, Kaye JA, Zhou Y, Pan C, Yi E, Eng J, Wang Q, Aebersold RH and Montine TJ. Quantitative proteomics of cerebrospinal fluid from patients with Alzheimer disease. J Alzheimers Dis. 2005; 7:125-133; discussion 173-180.

24. Abdi F, Quinn JF, Jankovic J, McIntosh M, Leverenz
JB, Peskind E, Nixon R, Nutt J, Chung K, Zabetian C, Samii A, Lin M, Hattan S, Pan C, Wang Y, Jin J, et al. Detection of biomarkers with a multiplex quantitative proteomic platform in cerebrospinal fluid of patients with neurodegenerative disorders. J Alzheimers Dis. 2006; 9:293-348

25. Tarawneh R, D’Angelo G, Macy E, Xiong C, Carter D, Cairns NJ, Fagan AM, Head D, Mintun MA, Ladenson JH, Lee JM, Morris JC and Holtzman DM. Visininlike protein-1: diagnostic and prognostic biomarker in Alzheimer disease. Ann Neurol. 2011; 70:274-285.

26. Mroczko B, Groblewska M, Zboch M, Muszynski P, Zajkowska A, Borawska R, Szmitkowski M, Kornhuber $\mathrm{J}$ and Lewczuk P. Evaluation of visinin-like protein 1 concentrations in the cerebrospinal fluid of patients with mild cognitive impairment as a dynamic biomarker of Alzheimer's disease. J Alzheimers Dis. 2015; 43:10311037.

27. Lin L, Long LK, Hatch MM and Hoffman DA. DPP6 domains responsible for its localization and function. J Biol Chem. 2014; 289:32153-32165.

28. Lin L, Sun W, Throesch B, Kung F, Decoster JT, Berner CJ, Cheney RE, Rudy B and Hoffman DA. DPP6 regulation of dendritic morphogenesis impacts hippocampal synaptic development. Nat Commun. 2013; 4:2270.

29. Wei Z, Sun M, Liu X, Zhang J and Jin Y. Rufy3, a protein specifically expressed in neurons, interacts with actinbundling protein Fascin to control the growth of axons. J Neurochem. 2014; 130:678-692.

30. Yamatani H, Kawasaki $\mathrm{T}$, Mita $\mathrm{S}$, Inagaki $\mathrm{N}$ and Hirata T. Proteomics analysis of the temporal changes in axonal proteins during maturation. Dev Neurobiol. 2010; 70:523537.

31. Cheng JS, Dubal DB, Kim DH, Legleiter J, Cheng IH, Yu GQ, Tesseur I, Wyss-Coray T, Bonaldo P and Mucke L. Collagen VI protects neurons against Abeta toxicity. Nat Neurosci. 2009; 12:119-121.

32. Kotlyar M, Pastrello C, Pivetta F, Lo Sardo A, Cumbaa C, Li H, Naranian T, Niu Y, Ding Z, Vafaee F, BroackesCarter F, Petschnigg J, Mills GB, Jurisicova A, Stagljar I, Maestro R, et al. In silico prediction of physical protein interactions and characterization of interactome orphans. Nat Methods. 2015; 12:79-84.

33. Cui Z, Tao T, Cheng C, Yang J, Shen Q, Ji Y, Li X, Liu $\mathrm{H}$, Shen A and Lu X. SSeCKS promote beta-amyloidinduced PC12 cells neurotoxicity by up-regulating tau phosphorylation in Alzheimer's disease. Mol Cell Biochem. 2010; 340:257-263.

34. Gozal YM, Duong DM, Gearing M, Cheng D, Hanfelt JJ, Funderburk C, Peng J, Lah JJ and Levey AI. Proteomics analysis reveals novel components in the detergentinsoluble subproteome in Alzheimer's disease. J Proteome Res. 2009; 8:5069-5079.

35. Doty RL. Olfactory dysfunction in Parkinson disease. Nat 
Rev Neurol. 2012; 8:329-339.

36. Hoyles K and Sharma JC. Olfactory loss as a supporting feature in the diagnosis of Parkinson's disease: a pragmatic approach. J Neurol. 2013; 260:2951-2958.

37. Alves J, Petrosyan A and Magalhaes R. Olfactory dysfunction in dementia. World J Clin Cases. 2014; 2:661667.

38. Gray AJ, Staples V, Murren K, Dhariwal A and Bentham P. Olfactory identification is impaired in clinic-based patients with vascular dementia and senile dementia of Alzheimer type. Int J Geriatr Psychiatry. 2001; 16:513-517.

39. Zhu YY, Ni DF and Xu CM. Gene expression profiles in the olfactory bulb from a rat model of Alzheimer's disease. J Alzheimers Dis. 2009; 18:581-593.

40. Masurkar AV and Devanand DP. Olfactory Dysfunction in the Elderly: Basic Circuitry and Alterations with Normal Aging and Alzheimer's Disease. Curr Geriatr Rep. 2014; 3:91-100.

41. Korolainen MA, Nyman TA, Aittokallio T and Pirttila T. An update on clinical proteomics in Alzheimer's research. J Neurochem. 2010; 112:1386-1414.

42. Brinkmalm A, Portelius E, Ohrfelt A, Brinkmalm G, Andreasson U, Gobom J, Blennow K and Zetterberg $\mathrm{H}$. Explorative and targeted neuroproteomics in Alzheimer's disease. Biochim Biophys Acta. 2015; 1854:769-778.

43. Krzyzanowska A, Garcia-Consuegra I, Pascual C, Antequera D, Ferrer I and Carro E. Expression of regulatory proteins in choroid plexus changes in early stages of Alzheimer disease. J Neuropathol Exp Neurol. 2015; 74:359-369.

44. Chang RY, Etheridge N, Nouwens AS and Dodd PR. SWATH analysis of the synaptic proteome in Alzheimer's disease. Neurochem Int. 2015; 87:1-12.

45. Unwin RD, Griffiths JR and Whetton AD. Simultaneous analysis of relative protein expression levels across multiple samples using iTRAQ isobaric tags with 2D nano LC-MS/ MS. Nat Protoc. 2010; 5:1574-1582.

46. Fernandez-Irigoyen J, Labarga A, Zabaleta A, de Morentin XM, Perez-Valderrama E, Zelaya MV and Santamaria E. Toward defining the anatomo-proteomic puzzle of the human brain: An integrative analysis. Proteomics Clin Appl. 2015; 9:796-807.

47. Kiddle SJ, Sattlecker M, Proitsi P, Simmons A, Westman E, Bazenet C, Nelson SK, Williams S, Hodges A, Johnston C, Soininen H, Kloszewska I, Mecocci P, Tsolaki M, Vellas $\mathrm{B}$, Newhouse S, et al. Candidate blood proteome markers of Alzheimer's disease onset and progression: a systematic review and replication study. J Alzheimers Dis. 2014; 38:515-531.

48. Bobkova NV, Garbuz DG, Nesterova I, Medvinskaya N, Samokhin A, Alexandrova I, Yashin V, Karpov V, Kukharsky MS, Ninkina NN, Smirnov AA, Nudler E and Evgen'ev M. Therapeutic effect of exogenous hsp70 in mouse models of Alzheimer's disease. J Alzheimers Dis.
2014; 38:425-435.

49. Kohli BM, Pflieger D, Mueller LN, Carbonetti G, Aebersold R, Nitsch RM and Konietzko U. Interactome of the amyloid precursor protein APP in brain reveals a protein network involved in synaptic vesicle turnover and a close association with Synaptotagmin-1. J Proteome Res. 2012; 11:40754090.

50. Hosp F, Vossfeldt H, Heinig M, Vasiljevic D, Arumughan A, Wyler E, Landthaler M, Hubner N, Wanker EE, Lannfelt L, Ingelsson M, Lalowski M, Voigt A and Selbach M. Quantitative interaction proteomics of neurodegenerative disease proteins. Cell Rep. 2015; 11:1134-1146.

51. Franceschini A, Szklarczyk D, Frankild S, Kuhn M, Simonovic M, Roth A, Lin J, Minguez P, Bork P, von Mering C and Jensen LJ. STRING v9.1: protein-protein interaction networks, with increased coverage and integration. Nucleic Acids Res. 2013; 41:D808-815.

52. Huang da W, Sherman BT and Lempicki RA. Systematic and integrative analysis of large gene lists using DAVID bioinformatics resources. Nat Protoc. 2009; 4:44-57.

53. Berson A, Barbash S, Shaltiel G, Goll Y, Hanin G, Greenberg DS, Ketzef M, Becker AJ, Friedman A and Soreq H. Cholinergic-associated loss of hnRNP-A/B in Alzheimer's disease impairs cortical splicing and cognitive function in mice. EMBO Mol Med. 2012; 4:730-742.

54. Donev R, Newall A, Thome J and Sheer D. A role for SC35 and hnRNPA1 in the determination of amyloid precursor protein isoforms. Mol Psychiatry. 2007; 12:681-690.

55. Obrdlik P, Diekert K, Watzke N, Keipert C, Pehl U, Brosch C, Boehm N, Bick I, Ruitenberg M, Volknandt W and Kelety B. Electrophysiological characterization of ATPases in native synaptic vesicles and synaptic plasma membranes. Biochem J. 2010; 427:151-159.

56. Qureshi HY, Li T, MacDonald R, Cho CM, Leclerc N and Paudel HK. Interaction of 14-3-3zeta with microtubuleassociated protein tau within Alzheimer's disease neurofibrillary tangles. Biochemistry. 2013; 52:6445-6455.

57. Sluchanko NN and Gusev NB. Probable participation of 14-3-3 in tau protein oligomerization and aggregation. J Alzheimers Dis. 2011; 27:467-476.

58. Pollerberg GE, Thelen K, Theiss MO and Hochlehnert BC. The role of cell adhesion molecules for navigating axons: density matters. Mech Dev. 2013; 130:359-372.

59. Djogo N, Jakovcevski I, Muller C, Lee HJ, Xu JC, Jakovcevski M, Kugler S, Loers G and Schachner M. Adhesion molecule L1 binds to amyloid beta and reduces Alzheimer's disease pathology in mice. Neurobiol Dis. 2013; 56:104-115.

60. Shetty A, Sytnyk V, Leshchyns'ka I, Puchkov D, Haucke $\mathrm{V}$ and Schachner M. The neural cell adhesion molecule promotes maturation of the presynaptic endocytotic machinery by switching synaptic vesicle recycling from adaptor protein 3 (AP-3)- to AP-2-dependent mechanisms. J Neurosci. 2013; 33:16828-16845. 
61. Zhou XH, Brakebusch C, Matthies H, Oohashi T, Hirsch E, Moser M, Krug M, Seidenbecher CI, Boeckers TM, Rauch U, Buettner R, Gundelfinger ED and Fassler R. Neurocan is dispensable for brain development. Mol Cell Biol. 2001; 21:5970-5978.

62. Brakebusch C, Seidenbecher CI, Asztely F, Rauch U, Matthies H, Meyer H, Krug M, Bockers TM, Zhou X, Kreutz MR, Montag D, Gundelfinger ED and Fassler R. Brevican-deficient mice display impaired hippocampal CA1 long-term potentiation but show no obvious deficits in learning and memory. Mol Cell Biol. 2002; 22:7417-7427.

63. Friedland-Leuner K, Stockburger C, Denzer I, Eckert GP and Muller WE. Mitochondrial dysfunction: cause and consequence of Alzheimer's disease. Prog Mol Biol Transl Sci. 2014; 127:183-210.

64. Moran M, Moreno-Lastres D, Marin-Buera L, Arenas J, Martin MA and Ugalde C. Mitochondrial respiratory chain dysfunction: implications in neurodegeneration. Free Radic Biol Med. 2012; 53:595-609.

65. Tillement L, Lecanu L and Papadopoulos V. Alzheimer's disease: effects of beta-amyloid on mitochondria. Mitochondrion. 2011; 11:13-21.

66. Luo X, Hou L, Shi H, Zhong X, Zhang Y, Zheng D, Tan Y, Hu G, Mu N, Chan J, Chen X, Fang Y, Wu F, He H and Ning Y. CSF levels of the neuronal injury biomarker visinin-like protein-1 in Alzheimer's disease and dementia with Lewy bodies. J Neurochem. 2013; 127:681-690.

67. Tarawneh R, Lee JM, Ladenson JH, Morris JC and Holtzman DM. CSF VILIP-1 predicts rates of cognitive decline in early Alzheimer disease. Neurology. 2012; 78:709-719.

68. Braunewell K, Riederer P, Spilker C, Gundelfinger ED, Bogerts $\mathrm{B}$ and Bernstein HG. Abnormal localization of two neuronal calcium sensor proteins, visinin-like proteins (vilips)-1 and -3, in neocortical brain areas of Alzheimer disease patients. Dement Geriatr Cogn Disord. 2001; 12:110-116.

69. Braunewell KH. The visinin-like proteins VILIP-1 and VILIP-3 in Alzheimer's disease-old wine in new bottles. Front Mol Neurosci. 2012; 5:20.

70. Schnurra I, Bernstein HG, Riederer $\mathrm{P}$ and Braunewell $\mathrm{KH}$. The neuronal calcium sensor protein VILIP-1 is associated with amyloid plaques and extracellular tangles in Alzheimer's disease and promotes cell death and tau phosphorylation in vitro: a link between calcium sensors and Alzheimer's disease? Neurobiol Dis. 2001; 8:900-909.

71. Bezprozvanny I and Mattson MP. Neuronal calcium mishandling and the pathogenesis of Alzheimer's disease. Trends Neurosci. 2008; 31:454-463.

72. An WF, Bowlby MR, Betty M, Cao J, Ling HP, Mendoza G, Hinson JW, Mattsson KI, Strassle BW, Trimmer JS and Rhodes KJ. Modulation of A-type potassium channels by a family of calcium sensors. Nature. 2000; 403:553-556.

73. McNicholas K, Chen $\mathrm{T}$ and Abbott CA. Dipeptidyl peptidase (DP) 6 and DP10: novel brain proteins implicated in human health and disease. Clin Chem Lab Med. 2009; 47:262-267.

74. Sun W, Maffie JK, Lin L, Petralia RS, Rudy B and Hoffman DA. DPP6 establishes the A-type $\mathrm{K}(+)$ current gradient critical for the regulation of dendritic excitability in CA1 hippocampal neurons. Neuron. 2011; 71:1102-1115.

75. Mori T, Wada T, Suzuki T, Kubota $Y$ and Inagaki N. Singar1, a novel RUN domain-containing protein, suppresses formation of surplus axons for neuronal polarity. J Biol Chem. 2007; 282:19884-19893.

76. Kitagishi Y and Matsuda S. RUFY, Rab and Rap Family Proteins Involved in a Regulation of Cell Polarity and Membrane Trafficking. Int J Mol Sci. 2013; 14:6487-6498.

77. Nagayama S, Homma $R$ and Imamura F. Neuronal organization of olfactory bulb circuits. Front Neural Circuits. 2014; 8:98.

78. Braak H, Alafuzoff I, Arzberger T, Kretzschmar H and Del Tredici K. Staging of Alzheimer disease-associated neurofibrillary pathology using paraffin sections and immunocytochemistry. Acta Neuropathol. 2006; 112:389404.

79. Alafuzoff I, Arzberger T, Al-Sarraj S, Bodi I, Bogdanovic N, Braak H, Bugiani O, Del-Tredici K, Ferrer I, Gelpi E, Giaccone G, Graeber MB, Ince P, Kamphorst W, King A, Korkolopoulou P, et al. Staging of neurofibrillary pathology in Alzheimer's disease: a study of the BrainNet Europe Consortium. Brain Pathol. 2008; 18:484-496.

80. Montine TJ, Phelps CH, Beach TG, Bigio EH, Cairns NJ, Dickson DW, Duyckaerts C, Frosch MP, Masliah E, Mirra SS, Nelson PT, Schneider JA, Thal DR, Trojanowski JQ, Vinters HV and Hyman BT. National Institute on AgingAlzheimer's Association guidelines for the neuropathologic assessment of Alzheimer's disease: a practical approach. Acta Neuropathol. 2012; 123:1-11.

81. Thal DR, Rub U, Orantes M and Braak H. Phases of A beta-deposition in the human brain and its relevance for the development of AD. Neurology. 2002; 58:1791-1800.

82. Mirra SS, Hart MN and Terry RD. Making the diagnosis of Alzheimer's disease. A primer for practicing pathologists. Arch Pathol Lab Med. 1993; 117:132-144.

83. Crary JF, Trojanowski JQ, Schneider JA, Abisambra JF, Abner EL, Alafuzoff I, Arnold SE, Attems J, Beach TG, Bigio EH, Cairns NJ, Dickson DW, Gearing M, Grinberg LT, Hof PR, Hyman BT, et al. Primary age-related tauopathy (PART): a common pathology associated with human aging. Acta Neuropathol. 2014; 128:755-766.

84. McKeith IG, Dickson DW, Lowe J, Emre M, O'Brien JT, Feldman H, Cummings J, Duda JE, Lippa C, Perry EK, Aarsland D, Arai H, Ballard CG, Boeve B, Burn DJ, Costa $D$, et al. Diagnosis and management of dementia with Lewy bodies: third report of the DLB Consortium. Neurology. 2005; 65:1863-1872.

85. Mackenzie IR, Neumann M, Baborie A, Sampathu DM, 
Du Plessis D, Jaros E, Perry RH, Trojanowski JQ, Mann $\mathrm{DM}$ and Lee VM. A harmonized classification system for FTLD-TDP pathology. Acta Neuropathol. 2011; 122:111113.

86. Roman GC, Tatemichi TK, Erkinjuntti T, Cummings JL, Masdeu JC, Garcia JH, Amaducci L, Orgogozo JM, Brun A, Hofman A and et al. Vascular dementia: diagnostic criteria for research studies. Report of the NINDS-AIREN International Workshop. Neurology. 1993; 43:250-260.

87. Litvan I, Hauw JJ, Bartko JJ, Lantos PL, Daniel SE, Horoupian DS, McKee A, Dickson D, Bancher C, Tabaton $\mathrm{M}$, Jellinger K and Anderson DW. Validity and reliability of the preliminary NINDS neuropathologic criteria for progressive supranuclear palsy and related disorders. J Neuropathol Exp Neurol. 1996; 55:97-105.

88. Kovacs T, Cairns NJ and Lantos PL. beta-amyloid deposition and neurofibrillary tangle formation in the olfactory bulb in ageing and Alzheimer's disease. Neuropathol Appl Neurobiol. 1999; 25:481-491.

89. Shilov IV, Seymour SL, Patel AA, Loboda A, Tang WH, Keating SP, Hunter CL, Nuwaysir LM and Schaeffer DA. The Paragon Algorithm, a next generation search engine that uses sequence temperature values and feature probabilities to identify peptides from tandem mass spectra. Mol Cell Proteomics. 2007; 6:1638-1655.

90. Liechtenstein T, Perez-Janices N, Gato M, Caliendo F, Kochan G, Blanco-Luquin I, Van der Jeught K, Arce F, Guerrero-Setas D, Fernandez-Irigoyen J, Santamaria E, Breckpot K and Escors D. A highly efficient tumorinfiltrating MDSC differentiation system for discovery of anti-neoplastic targets, which circumvents the need for tumor establishment in mice. Oncotarget. 2014; 5:78437857.

91. Tang WH, Shilov IV and Seymour SL. Nonlinear fitting method for determining local false discovery rates from decoy database searches. J Proteome Res. 2008; 7:36613667.

92. Croft D, Mundo AF, Haw R, Milacic M, Weiser J, Wu G, Caudy M, Garapati P, Gillespie M, Kamdar MR, Jassal B, Jupe S, Matthews L, May B, Palatnik S, Rothfels K, et al. The Reactome pathway knowledgebase. Nucleic Acids Res. 2014; 42:D472-477. 